\title{
Insights into the Acadian orogeny, New England Appalachians: a provenance study of the Carrabassett and Kittery formations, Maine
}

\author{
Michael J. Dorais ${ }^{*}$, Robert P. Wintsch ${ }^{2}$, Wendy R. Nelson ${ }^{3}$, and Michael Tubrett ${ }^{4}$ \\ 1. Department of Geological Sciences, Brigham Young University, Provo, Utah 84602, USA \\ 2. Department of Geological Sciences, Indiana University, Bloomington, Indiana 47405, USA \\ 3. Department of Geosciences, Penn State University, University Park, Pennsylvania 16802, USA \\ 4. CREAIT Network, MicroAnalysis Facility, Inco Innovation Centre (MAF-IIC), \\ Memorial University, St. John's, Newfoundland A1B 3X5, Canada \\ *Corresponding author:<dorais@byu.edu>
}

Date received: 07 July 2008 I Date accepted: 11 February 2009

\begin{abstract}
The Central Maine Basin and Merrimack Trough are Silurian basins that formed adjacent to or were accreted to the Laurentian margin during the Acadian orogeny. The Early Devonian Carrabassett Formation of the Central Maine Basin and the Kittery Formation of the Merrimack Trough have major and trace element compositions indicative of a passive continental margin provenance, not unlike the older formations of the Central Maine Basin that are thought to have been derived from Laurentian sources. However, both the Carrabassett and Kittery formations have paleocurrent indicators of outboard sources. The Carrabassett Formation is one of the youngest formations of the Central Maine Basin and was deposited just prior to the Acadian orogeny. The Carrabassett and Kittery formations have major and trace element concentrations suggestive of passive margin turbidites derived from intermediate to felsic sources, inconsistent with a juvenile Avalonian provenance. The Carrabassett Formation contains detrital zircon grains that match the ages of peri-Gondwanan Ganderia. Unlike the dominance of positive bulk-rock $\varepsilon_{\mathrm{Nd}}$ values that are characteristic of Avalonia, Ganderia has negative $\varepsilon_{\mathrm{Nd}}$ values that are a better match for the negative $\varepsilon_{\mathrm{Nd}}$ values of the Carrabassett and Kittery formations. However, Ganderia accreted to Laurentia during the Salinic orogeny, prior to the deposition of the Carrabassett Formation, and was basement to the sediments of the Central Maine Basin upon which the Carrabassett and other formations were deposited. Wedging of Ganderia by Avalonia during the initial stages of the Acadian orogeny may have uplifted Ganderia, forming highlands outboard of the Central Maine Basin that served as the source of the Carrabassett Formation sediments.
\end{abstract}

\section{RÉSUMÉ}

Le bassin central du Maine et la cuvette de Merrimack constituent des bassins siluriens s'étant formés le long de la marge laurentienne ou s'y étant accrétés au cours de l'orogenèse acadienne. La Formation du Dévonien précoce de Carrabassett, dans le bassin central du Maine, et la Formation de Kittery, de la cuvette de Merrimack, présentent des compositions en éléments majeurs et traces signalant une provenance d'une marge continentale passive, à l'instar des formations plus âgées du bassin central du Maine qu'on pense originaires de sources laurentiennes. Les formations de Carrabassett et de Kittery comportent toutefois des indicateurs de paléocourants de sources extérieures. La Formation de Carrabassett constitue l'une des formations les plus récentes du bassin central du Maine; elle s'est mise en place juste avant l'orogenèse acadienne. Les caractéristiques géochimiques et géochronologique des formations de Carrabassett et de Kittery pourraient par conséquent permettre l'identification du terrane de collision. Les formations de Carrabassett et de Kittery possèdent des concentrations d'éléments majeurs et traces évoquant les turbidites de marge passive en provenance de sources intermédiaires à felsiques, ce qui est contradictoire avec une origine avalonienne juvénile. La Formation de Carrabassett comporte des grains détritiques de zircon correspondant aux âges du Ganderia périgondwanien. Contrairement à la prédominance de concentrations $\varepsilon_{\mathrm{Nd}}$ positives de roche en vrac caractéristiques d'Avalonia, Ganderia présentent des concentrations $\varepsilon_{\mathrm{Nd}}$ négatives qui cadrent mieux avec les concentrations $\varepsilon_{\mathrm{Nd}}$ négatives des formations de Carrabassett et de Kittery. Ganderia s'est toutefois accrété à Laurentia au cours de l'orogenèse salinique, avant le dépôt de la Formation de Carrabassett, et il a constitué le socle des sédiments du bassin central du Maine sur lesquels Carrabassett et d'autres formations se sont déposées. L'enfoncement d'Avalonia sous Ganderia au cours des stades initiaux de l'orogenèse acadienne pourrait avoir soulevé Ganderia, formant un massif à l'extérieur du bassin central du Maine qui a servi de source aux sédiments de la Formation de Carrabassett.

[Traduit par la redaction] 


\section{INTRODUCTION}

More studies have been conducted of and more has been written about the Acadian orogeny than any other New England orogenic event (e.g., Osberg et al. 1989; Roy and Skehan 1993; Rankin 1994; Bradley et al. 2000; Eusden et al. 2000; Tucker et al. 2001 and references therein). In spite of this volume of work, the fundamental problem of what caused the New England part of the Acadian orogeny remains.

The orogenies of the New England Appalachians resulted from the collision of several microcontinents to the Laurentian margin. In the context of this study, the most significant microcontinents are Ganderia and Avalonia. From the early to middle Paleozoic, the Laurentian margin progressively expanded due to the accretion of these microcontinents. They represent two separate peri-Gondwanan blocks that had separate but similar Neoproterozoic histories but were distinct from the Early Paleozoic (van Staal 2007). Ganderia was a Late Neoproterozoic to Early Cambrian arc terrane that rifted off Amazonia at about $505 \mathrm{Ma}$ whereas Avalonia, a largely juvenile arc unconformably covered by Cambrian-Ordovician platform sediments, probably rifted from Gondwana 30 million years later (van Staal 2007). The Salinic orogeny was caused by collision of the Ganderian margin and Laurentian at 430-422 Ma. Many geologists think that the subsequent collision of Avalonia with composite Laurentia at 420-400 Ma caused the Acadian orogeny (e.g., Osberg et al. 1989; Robinson et al. 1998), but lack of evidence of Acadian metamorphism in the New England part of Avalonia (Fig. 1) is a challenge to this interpretation (Walsh et al. 2007; Aleinikoff et al.2007). Instead, the geochronology of Avalonia and inboard peri-Gondwanan terranes document a Pennsylvanian-Late Permian orogenic event (Eusden and Barreiro 1988; Spear and Harrison 1989; Tucker and Robinson 1990; Dallmeyer and Takasu 1992; Wintsch et al. 1992; Getty and Gromet 1992; West 1993; Lux and West 1993; Walsh et al. 2007).

West and north of the New England Avalonia are the peri-Gondwanan Willimantic and Pelham domes and the Massabesic Gneiss Complex (Fig. 1). In common with Avalonia, no Acadian metamophic or igneous ages have been identified in the Willimantic or the Pelham domes. Both domes contain orthogneisses with ages of $\sim 620 \mathrm{Ma}$, a common age of peri-Gondwanan rocks, with a strong overprinting of a $\sim 280$ Ma event in the Alleghanian. As acknowledged by Robinson

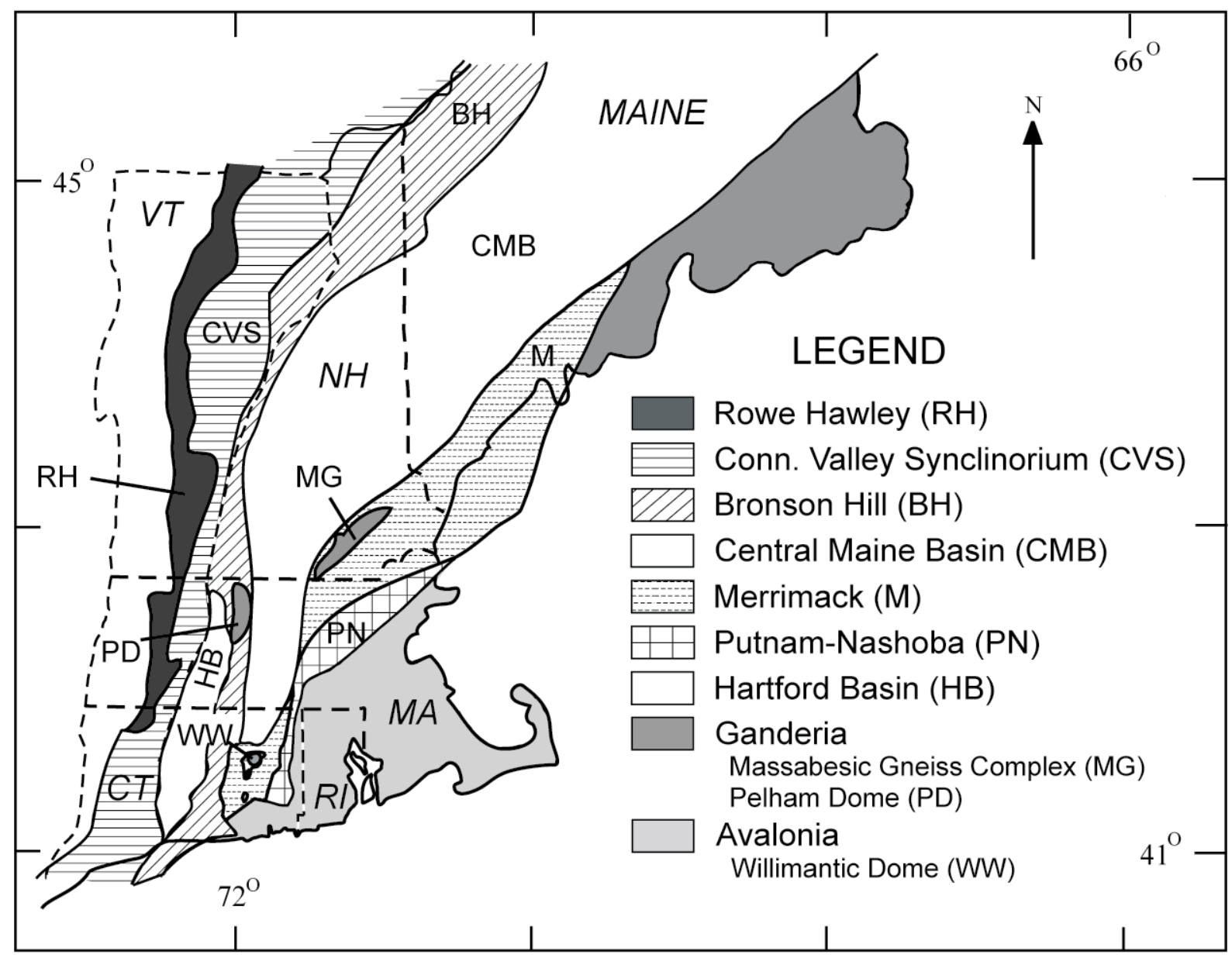

Fig. 1. Generalized geologic map showing the locations of the lithotectonic zones of New England (after Wintsch et al. 1992). The Carrabassett Formation is located in the Central Maine Basin (CMB). 
et al. (1998), this lack of Ordovician-Devonian igneous activity and Silurian-Devonian metamorphism is inconsistent with the assignment of these domes to a peri-Gondwanan terrane that collided with Laurentia prior to the Alleghanian. For these reasons, some workers think that Avalonia did not arrive in southern New England until the Alleghanian orogeny (Zartman and Naylor 1984; Wintsch and Sutter 1986; Mosher et al. 1993; Wintsch et al. 1993; Rankin 1994; Walsh et al. 2007) and may have been emplaced along strike-slip faults in the Late Paleozoic (Robinson et al. 1998). Alternatively, Wintsch et al. (1992) suggested that the portion of Avalonia that collided during the Acadian was subsequently thrust further under the allochthonous rocks of the Central Maine Basin, Merrimack Trough, and Putnam-Nashoba terrane during the Alleghanian orogeny and is no longer exposed to provide evidence of Avalonian involvement with the Acadian event.

The Massabesic Gneiss complex shares the same 620 and $280 \mathrm{Ma}$ events with Avalonia of southeastern New England (Aleinikoff et al. 1979), and is the only peri-Gondwanan inlier that may preserve evidence of the Acadian orogeny. This gneiss contains amphibolite and an attenuated granitic dike with $\sim 400 \mathrm{Ma}$ zircon and monazite, respectively (Aleinikoff $e t$ al. 1995). The possibility that the Massabesic Gneiss Complex is of Ganderian affinity (Moench and Alienikoff 2003; Dorais et al. 2007) suggests that the complex had docked with Laurentia in the Salinic orogeny and, rather than having been the Acadian colliding terrane, it was on the receiving end of Avalonia's collision during the Acadian. Thus the question remains: What lithotectonic elements collided with Laurentia in this part of the New England Appalachians to cause the Acadian orogeny?

A Silurian to Early Devonian basin called the Central Maine Basin developed between Laurentia and the approaching terrane that docked to cause the Acadian orogeny. The earliest sediments deposited in the Central Maine Basin were derived from Laurentia whereas the uppermost formations preserve paleocurrent indicators from the east, suggesting an outboard source thought to be Avalonia (Hanson and Bradley 1989; Hanson et al. 1993; Bradley and Hanson 2002). Because sediments carry the major and trace element, isotopic, and detrital zircon signatures of their source rocks (O'Nions et al. 1983; Allegre and Rousseau 1984; Michard et al. 1985; McLennan et al. 1990; Gleason et al. 1994; Anderson and Sampson 1995; Bock et al.1998; Wintsch et al.2007), we undertook this study to determine whether or not the bulk-rock geochemical characteristics and detrital zircon geochronology of easterly derived sediments, i.e., the Carrabassett Formation of Maine, would identify the outboard terrane that collided with Laurentia. We compare these geochemical and geochronological parameters with those of the older formations of the Central Maine Basin in order to make comparisons between the source regions of sediments shed from Laurentia and outboard sources.

The Silurian Merrimack Trough lies to the east, outboard of the Central Maine Basin (Fig.1). This terrane is also an enigmatic belt that has traditionally been interpreted as periGondwanan (e.g., Hibbard et al. 2006). Like the Carrabassett Formation of the Central Maine Basin, the Kittery Formation of the Merrimack Trough has paleocurrent indicators of an easterly source (Rickerich 1983). We also compare the bulkrock geochemical, isotopic, and detrital zircon characteristics of the Kittery Formation with those of the Carrabassett Formation in order to evaluate provenance characteristics of the approaching Acadian colliding element.

\section{GEOLOGIC SETTING}

\section{Central Maine Basin}

The formations of the Central Maine Basin (Fig. 1) have been given different names by various workers depending on the region of study, but because we concentrated on the formations of western Maine, we use the names established by Moench and co-workers (e.g., Moench and Pankiwskyj 1988). We specifically focused on the Silurian and Devonian rocks of the Central Maine Basin which span a time frame from significantly before to just prior to and perhaps concurrent with the initial deformation of the Acadian orogeny (Bradley and Hanson 2002). From oldest to youngest, the formations of interest are the Rangeley, Perry Mountain, Smalls Falls, Madrid, and Carrabassett.

These formations are draped across what has been called a tectonic hinge that coincides with the eastern margin of the Bronson Hill anticlinorium (Fig. 1; Hatch et al. 1983). Northwest of the hinge, the sediments were deposited on a narrow shelf and lie unconformably on pre-Silurian rocks. Southeast of the hinge, the metasedimentary rocks thicken, representing the site of the Central Maine depositional basin. The hinge line appears to have been an active tectonic feature that controlled sedimentation from early Silurian to the culmination of the Acadian orogeny. The direction from which these sediments were derived is crucial to our study. An easterly or southeasterly transport direction is suggestive of Laurentian provenance. In contrast, westerly transportation directions have been interpreted as favoring a peri-Gondwanan source (Bradley and Hanson 2002).

The Rangeley Formation consists of three members, A, B, and C (Moench and Pankiwskyj 1988). Parts A and B thicken by an order of magnitude across the tectonic hinge to the southeast. Part A consists of a large sheet of marine sandstone and conglomerate, approximately $1200 \mathrm{~m}$ thick in the Rangeley quadrangle in Maine. The conglomerate includes boulders of the distinctive Ordovician Attean Quartz Monzonite from the Boundary Mountain anticlinorium to the northwest. Limited paleocurrent data suggest that the Perry Mountain and Smalls Falls formations were also derived from a source to the northwest (Hanson et al. 1993; Bradley and Hanson 2002). Interbedded quartzose sandstone and shale of the Perry Mountain Formation coarsen upward and to the northwest, also indicating a source from the northwest. Higher in the Silurian rocks of the Central Maine Basin, paleocurrent indicators indicate a non-western source area (Moench and Boudette 1970; Ludman and Griffin 1974; Ludman 1976; Bradley and 
Hanson 1989, 2002). Three observations indicate transportation and perhaps derivation of the Madrid Formation from the northeast (Hanson et al. 1993): (1) southeasterly paleocurrent indicators, (2) decreasing ratio of facies B to facies D turbidites to the southwest, and (3) thinning of the Madrid Formation to the southwest.

The Carrabassett Formation, correlative with the Littleton Formation of New Hampshire (Moench and Pankiwskyj 1988; Hatch and Moench 1984), is the youngest, most widespread pre-Acadian formation of the Central Maine Basin. It was deposited just prior to the onset of Acadian deformation and consists of $\sim 2000 \mathrm{~m}$ of Early Devonian, mud-rich turbidites that are locally metamorphosed to the chlorite zone of the greenschist facies (Hanson et al. 1993). Several facies are identified, from massive sandstones, undifferentiated thin to thick-bedded turbidites, chaotic facies, and thick-bedded turbidites to thin-bedded turbidites. The chaotic strata are considered to be olistrostromes. Coarse sandstone and conglomerate are absent. Paleocurrent data show a dominant flow from the southeast. Bradley and Hanson (2002) concluded that the combination of these facies and paleocurrent indicators reveals that the sediments were deposited on a submarine slope that descended to the northwest in the Central Maine depositional basin. High sedimentation rates, coupled with earthquakes from early Acadian tectonic disturbances, led to large amounts of sediment being remobilized and deposited as slumps and debris flows. As such, the Carrabassett Formation would represent deposition during the demise of the deep-water Central Maine Basin as it closed during the initial stages of Acadian collision.

\section{Merrimack Trough}

The Merrimack Trough is almost $400 \mathrm{~km}$ long, extending from Maine and southeastern New Hampshire through Massachusetts almost to Long Island Sound (Fig.1). In New Hampshire, it is in fault contact with the Massabesic Gneiss Complex on the northwest and with the Rye Complex on the southeast. It was originally defined by Katz (1917) to consist of the Kittery, Eliot, and Berwick formations that constitute a thick sequence of calcareous turbidites in the New Hampshire and Maine (Katz 1917; Billings 1956; Bothner et al. 1984; Bothner and Hussey 1999). Recently, Wintsch et al. (2007) concluded from detrital zircon geochronology that the Berwick Formation was Silurian and Laurentia-derived, whereas the Kittery Formation (middle Ordovician or younger), with its paleocurrent indicators of an eastern source and different detrital zircon signatures, is peri-Gondwanan. They interpreted the fault that separates the Berwick Formation from the Kittery and Eliot formations as a thrust fault that placed the Laurentiaderived Berwick on top of the peri-Gondwana-derived Kittery and Eliot. Thus it is particularly interesting to compare the provenance signature of the Carrabassett Formation with that of the Kittery Formation, as both appear to have an outboard provenance and have the potential to yield information about the Acadian colliding terrane.

\section{SAMPLE LOCATIONS}

Samples from the Carrabassett and Madrid formations were collected in Maine at stops 4, 6, 7, and 8 described in the Hanson et al. (1993) field guide. Rangeley Formation samples were collected in central New Hampshire at stops 1 and 2 of Lyons (1988) and stops 5, 7, and 9 of Eusden (1988). Berwick Formation samples were collected along a traverse starting at Exit 4 on Interstate 93 at Derry, New Hampshire extending eastward on Route 102 to Raymond, New Hampshire. The Eliot Formation was sampled at Stop VI-7 of Bothner (1989) at the junction of Route 155 and US4. Samples of the Kittery Formation were taken from Bothner's Stop VI-8 at Great Bay, New Hampshire, at Ogunquit, Moody Point in Wells, and at Cape Elizabeth, Maine. Approximate sample locations are shown on Figure 2.

\section{ANALYTICAL METHODS}

Detrital zircons were obtained from a ca. $5 \mathrm{~kg}$ sample of the Carrabassett Formation collected at Stop 4 of Hansen et al. (1993) at the Big Wilson Stream in Elliotsville, Maine. Zircons were extracted at Memorial University of Newfoundland using conventional mineral separation techniques (crushing, Wilfley table, and heavy liquids). Grains from the least magnetic split obtained with a Frantz isodynamic separator were hand picked in alcohol under a binocular microscope. The selected grains were mounted in an epoxy-filled grain mount and polished to obtain a flat surface. Bulk-rock XRF analyses were conducted on a Siemens SRS 303 instrument in the Department of Geological Sciences at Brigham Young University. Additional trace elements were analyzed by ICP-MS by ALS Chexex in Reno, Nevada.

In situ LA-ICP-MS analyses of areas within the selected crystals of zircon were carried out using a VG PlasmaQuad PQ-2 S+ instrument coupled to a NUWAVE UP213 nm NdYAG laser at Memorial University of Newfoundland. Additional zircons were analyzed with an Element XR coupled to a GEOLAS 193 $\mathrm{nm}$ eximer laser system following the methods of Košler et al. (2002). Raw data were corrected for electron multiplier dead time (20 ns) and processed off-line using an Excel spreadsheet program (LAMDATE) to integrate signals from each sequential set of 10 sweeps; the method follows that of Kosler et al. (2002). The measured ${ }^{207} \mathrm{~Pb} / 206 \mathrm{~Pb},{ }^{208} \mathrm{~Pb} /{ }^{206} \mathrm{~Pb},{ }^{206} \mathrm{~Pb} /{ }^{238} \mathrm{U}$, and ${ }^{207} \mathrm{~Pb} / 235 \mathrm{U}$ ratios were calculated and blank corrected for each analysis. The natural ${ }^{238} \mathrm{U} /{ }^{235} \mathrm{U}$ ratio of 137.88 was used to calculate the ${ }^{235} \mathrm{U}$ since it was not acquired with other isotopes due to its low natural abundance. Aspiration of the tracer solution allowed for a real-time instrument mass bias correction using known isotopic ratios of the tracer solution measured while the sample was ablated; this technique is largely independent of matrix effects that can variably influence measured isotopic ratios and hence the resulting ages (Kosler et al. 2002; Cox et al. 2003). The amount of common Pb present in the analyzed zircon grains was insignificant relative to the con- 


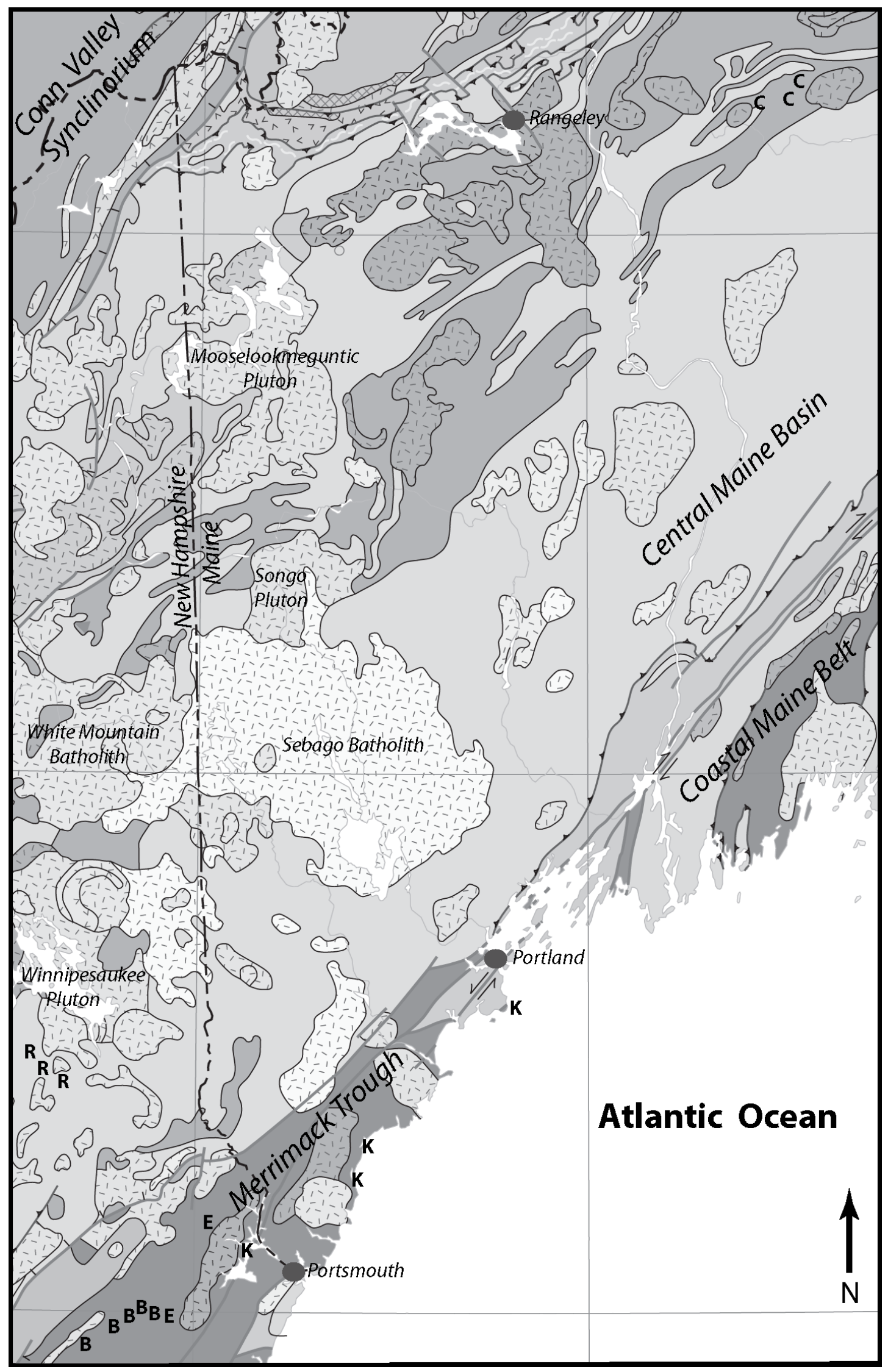

Fig. 2. Generalized geologic map of part of Maine and New Hampshire (from Hibbard et al. 2006) showing the approximate sample locations for the Carrabassett (C), Rangeley (R), Berwick (B), Eliot (E), and Kittery (K) formations. 
tent of radiogenic $\mathrm{Pb}$ and therefore, no common $\mathrm{Pb}$ correction was applied to the data. Accuracy and reproducibility of $\mathrm{U}-\mathrm{Pb}$ analysis were monitored by using zircon 91500 , a natural inhouse zircon standard with a known TIMS U-Pb age of 1065 $\pm 3 \mathrm{Ma}$ (Wiedenbeck et al. 1995). Final ages and concordia diagrams were produced using the Isoplot/Ex macro (Ludwig 2000) in conjunction with LAMDATE Excel spreadsheet program (Kosler et al. 2002).

\section{RESULTS}

\section{Bulk-rock Analyses}

Bulk-rock major and trace element analyses of the Carrabassett, Rangeley, Smalls Falls, and Madrid formations of the Central Maine Basin and the Kittery, Berwick and Eliot formations of the Merrimack Trough are given in Table 1 and sample locations in Appendix A. Before discussing these results, it is appropriate to discuss how well these metasedimentary rocks represent initial compositions and the potential of element mobility during diagenesis and metamorphism. Cullers et al. (1997) presented a major and trace element and $\mathrm{Nd}$ isotopic study of the Rangeley, Perry Mountain, and Smalls Falls formations of western Maine. Their objective was to test the commonly accepted notion that the REE, Th, Sc, Co, and $\mathrm{Cr}$ are the most immobile elements during weathering, diagenesis, and metamorphism and as such, can be used to determine the provenance of metasedimentary rocks. They found that most major and trace element abundances relative to $\mathrm{Al}_{2} \mathrm{O}_{3}$ are statistically identical between zones of the same formation as well as between formations, suggesting no mobilization of the elements of interest in these rocks. Systematic compositional variations were attributed to varying proportions of clay minerals, feldspars, and quartz in the original protoliths. Some samples of the Perry Mountain Formation, however, are anomalous in that they have low REE contents and unrealistically old $\mathrm{Nd}_{\mathrm{TDM}}$ model ages, suggestive of open system behavior. Bock et al. (2004) also found that anomalous REE patterns and old $\mathrm{Nd}_{\mathrm{TDM}}$ model ages of several formations of the northern Appalachians are indicative of open system behavior. Our samples show neither anomalous REE behavior nor unrealistically old $\mathrm{Nd}_{\mathrm{TDM}}$ model ages; hence, we assume that the REE, $\mathrm{Th}, \mathrm{Sc}, \mathrm{Co}$, and $\mathrm{Cr}$ concentrations permit determination of provenance characteristics as shown in numerous other studies (Bhatia and Crook 1986; Cox et al. 1995; Cullers 1994, Cullers et al. 1975, 1979, 1997; Wronkiewicz and Condie 1990).

Log values of $\mathrm{SiO}_{2} / \mathrm{Al}_{2} \mathrm{O}_{3}$ versus $\mathrm{Fe}_{2} \mathrm{O}_{3} / \mathrm{K}_{2} \mathrm{O}$ (Fig. 3) show that the samples (including analyses of Lathrop et al. 1996 and Cullers et al. 1997) plot as shale and wacke with a few samples scattering in the Fe-sandstone field (Herron 1988). The Rangeley Formation is dominated by shale protoliths whereas the other formations are wacke-dominated. Ague $(1991,1997)$ found evidence of $\mathrm{SiO}_{2}$ loss in the Littleton Formation with increasing metamorphic grade so the lower $\log \left(\mathrm{SiO}_{2} / \mathrm{Al}_{2} \mathrm{O}_{3}\right)$ values of the Rangeley Formation compared to the Carrabassett may be influenced by $\mathrm{SiO}_{2}$ loss during metamorphism.

Because compatible elements have higher concentrations

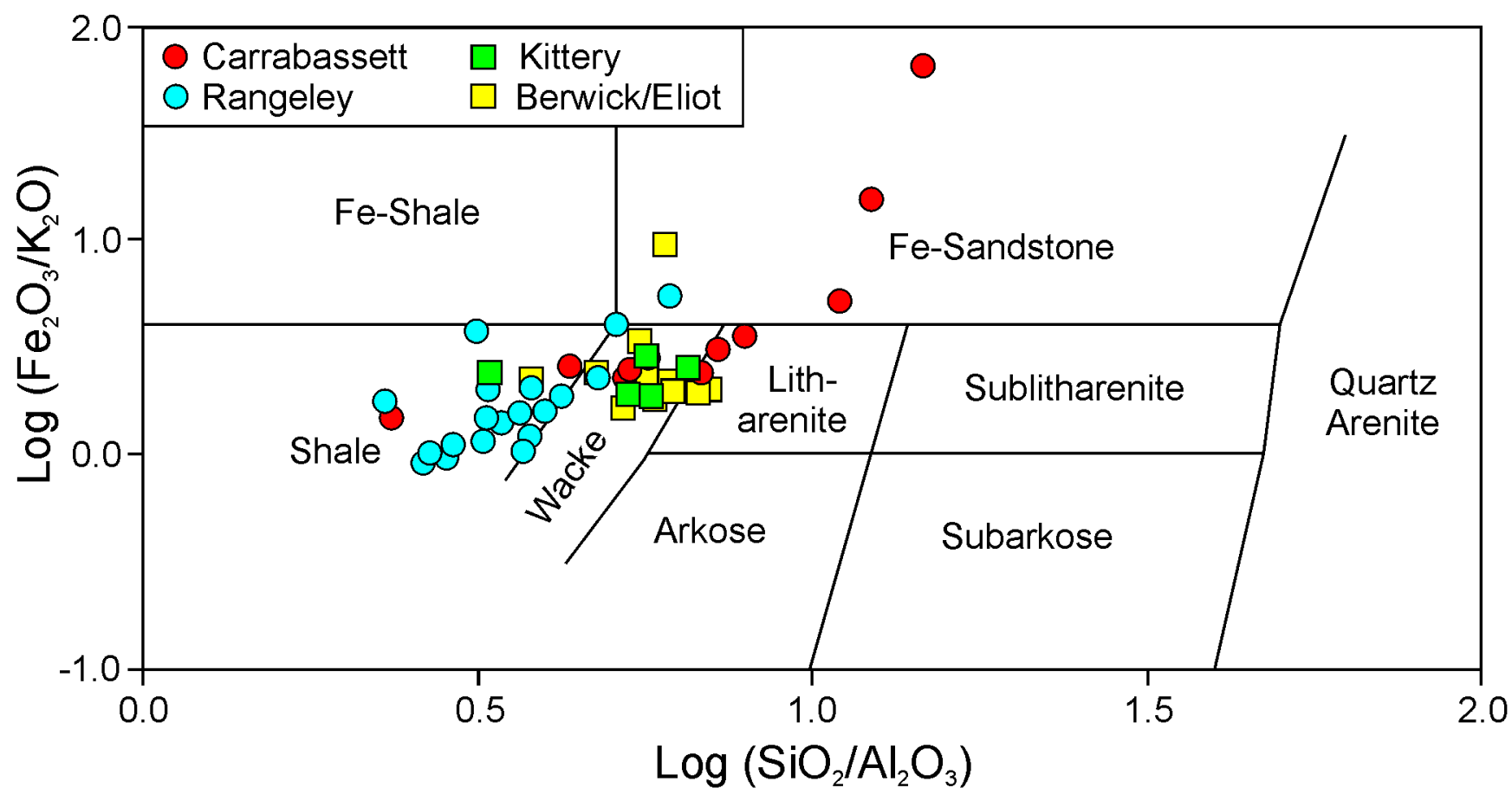

Fig. 3. $\mathrm{Log}\left(\mathrm{SiO}_{2} / \mathrm{Al}_{2} \mathrm{O}_{3}\right)$ versus $\log \left(\mathrm{Fe}_{2} \mathrm{O}_{3} / \mathrm{K}_{2} \mathrm{O}\right)$ classification diagram (after Herron 1988). Carrabassett Formation plot in fields from shale to Fe-sandstone. Most of the samples from the Kittery Formation and Berwick/Eliot formations plot as wacke. Our Rangeley Formation samples do not include the basal conglomeratic member and are primarily shale. 


\begin{tabular}{|c|c|c|c|c|c|c|}
\hline 芯 & $\beth^{\infty} \exists \simeq \simeq \stackrel{\sim}{a} \beth^{n}$ & 율 & 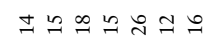 & $\exists \simeq 8$ & $\simeq \stackrel{2}{\simeq}$ & $\because 2$ 늠 \\
\hline 나 & 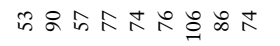 & ふฌ & in $38: 8$ 果 8 & 脶果夺 & 웍 8 in in in & 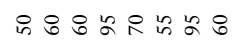 \\
\hline$z$ & 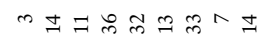 & 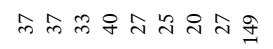 & $\because=\infty \Rightarrow$ in +9 & 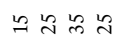 & 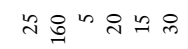 & 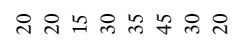 \\
\hline $\mathrm{z}$ & 可 F & 的的品东人和品子总 & 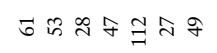 & 모웜 & 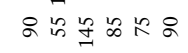 & 요요 \& \\
\hline 8 & 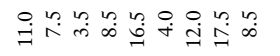 & 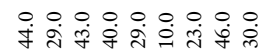 & 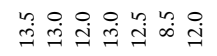 & 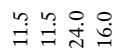 & 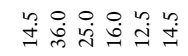 & 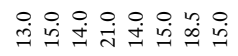 \\
\hline نे & 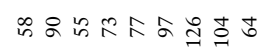 & 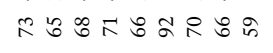 & 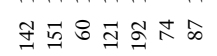 & & & \\
\hline & $\circ \stackrel{n}{\sim} \circ \sigma=\curvearrowleft \leadsto$ & 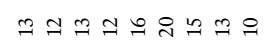 & $\ddot{\sim} \pm \pm \approx a m$ & 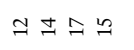 & $\simeq \cong 9 \cong \pm \cong$ & $\cong \pm \simeq \quad \cong \cong \mathscr{I}$ \\
\hline 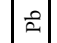 & 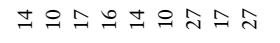 & $\pm \vec{N} \vec{N} \coprod_{\sim}^{\infty}[\vec{m} \vec{N} \pm$ & 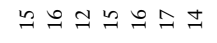 & 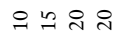 & R n n in 8 舟 & 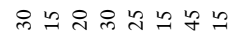 \\
\hline 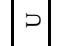 & & 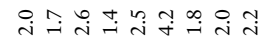 & & 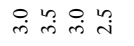 & 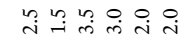 & 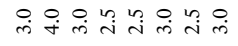 \\
\hline F & 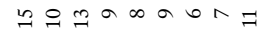 & 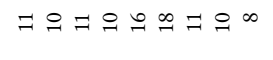 & $\infty \nexists^{\infty} \simeq N z^{n}$ & & $\infty \pi \approx \infty$ & $=ㄱ=\infty$ 의 $=0$ \\
\hline$\approx \pi$ & 610 & 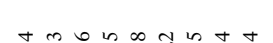 & $00 \mathrm{~N}$ & & $\begin{array}{ll}3 & 3 \\
0 & 0 \\
0 & =\end{array}$ & 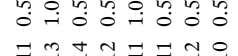 \\
\hline & & & & & $F+$ & $\Rightarrow 7$ \\
\hline & 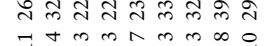 & 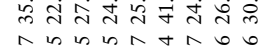 & $\begin{array}{ll}0 \\
0\end{array}$ & & & 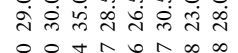 \\
\hline & & & & & & \\
\hline$=1$ & 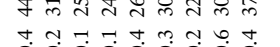 & 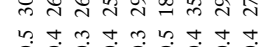 & men & & & \\
\hline 2 & 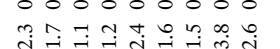 & 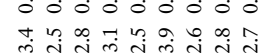 & 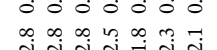 & $\therefore$ & 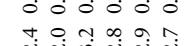 & \\
\hline घ & 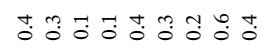 & 菅范范范 & 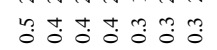 & ?. & ¿ & 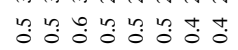 \\
\hline 苗 & 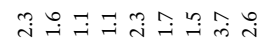 & 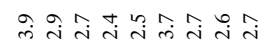 & 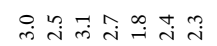 & j & बे $\overrightarrow{\text { iे }}$ & कु \\
\hline 원 & 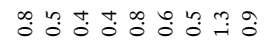 & अ & 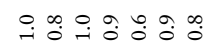 & $=8$ & 국: & 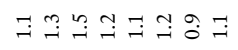 \\
\hline 定 & 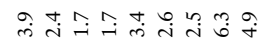 & 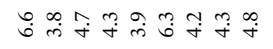 & 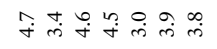 & 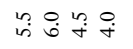 & 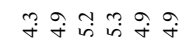 & 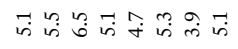 \\
\hline 至 & 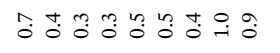 & 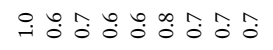 & $\dot{b}: \hat{0}$ & $\exists$ 경 & 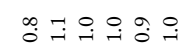 & 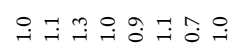 \\
\hline gु & 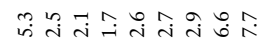 & 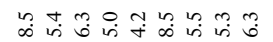 & 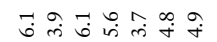 & 它总守字 & 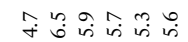 & ம் \\
\hline 可 & 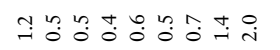 & 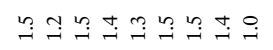 & 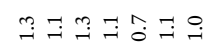 & $\rightarrow$ & 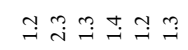 & 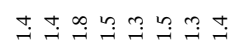 \\
\hline : & 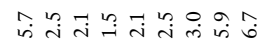 & 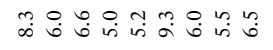 & 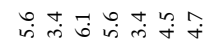 & $\rightarrow \infty$ & $\hat{\mathrm{n}}$ & : \\
\hline$\vec{z}$ & 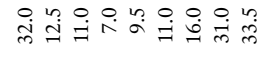 & 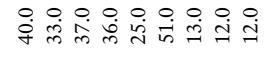 & कि & & D & 呙 \\
\hline 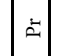 & 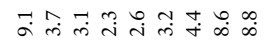 & 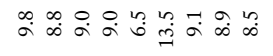 & 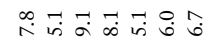 & 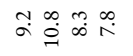 & 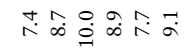 & 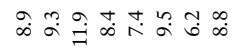 \\
\hline 8 & 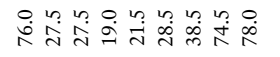 & $\begin{array}{ll}0 \\
0 \\
\infty \\
\infty\end{array}$ & 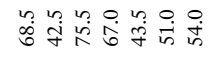 & ت. & 용 & 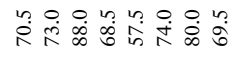 \\
\hline 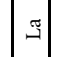 & 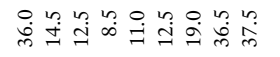 & $\underset{f}{+}$ & & $\stackrel{n}{m}$ & 車 & 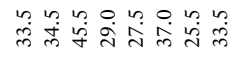 \\
\hline ๓ & 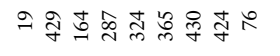 & 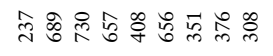 & 象赵 & 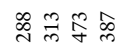 & 学嗯 & 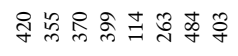 \\
\hline in & 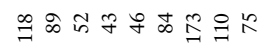 & 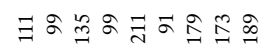 & ฮี ฏ & ? & $\stackrel{\partial}{a}$ & 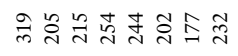 \\
\hline 3 & 유 ๙ & 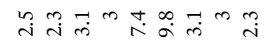 & 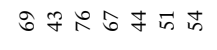 & 1 & สี & 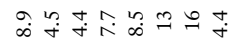 \\
\hline$\approx$ & 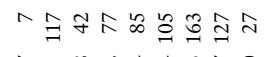 & 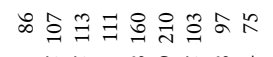 & 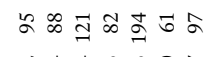 & 80 & 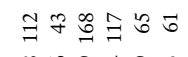 & ホ \\
\hline త్రీ & 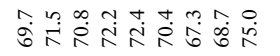 & 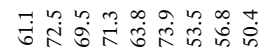 & 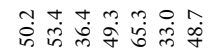 & in & 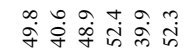 & 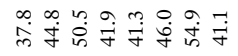 \\
\hline & 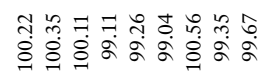 & 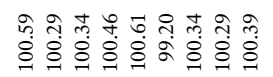 & 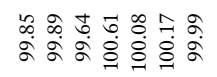 & & & 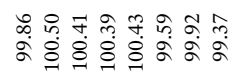 \\
\hline $\bar{\Xi}$ & 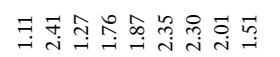 & 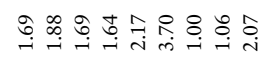 & 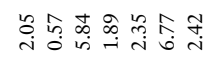 & 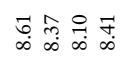 & 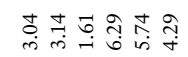 & ک્ગ \\
\hline 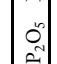 & 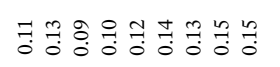 & mo & 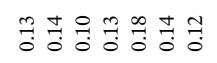 & $\stackrel{\infty}{2}$ & 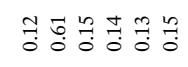 & 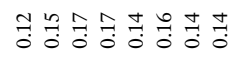 \\
\hline ○ & 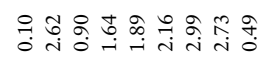 & 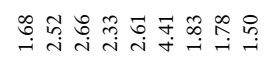 & ત̂: & 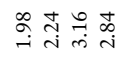 & 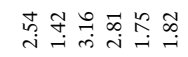 & 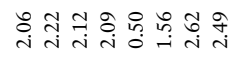 \\
\hline ชู & 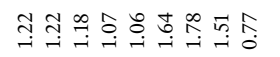 & 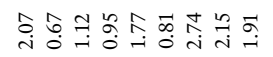 & 可 & $\stackrel{\infty}{\infty} \underset{\sim}{\infty} \stackrel{\infty}{\rightarrow}$ & 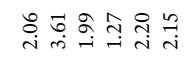 & 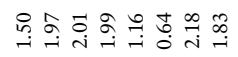 \\
\hline ల్ల & 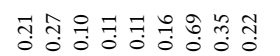 & 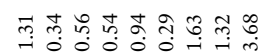 & 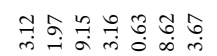 & 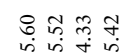 & 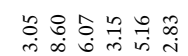 & 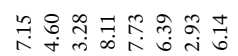 \\
\hline 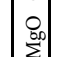 & $\hat{\hat{0}}$ & 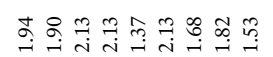 & 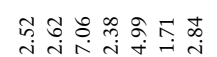 & 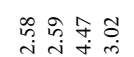 & 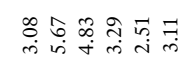 & 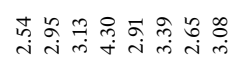 \\
\hline$\sum^{2}$ & $=$ & 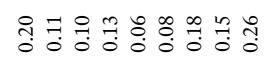 & 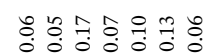 & $\hat{a}$ & 赵 & 궁형 \\
\hline & $\begin{array}{l}\mathcal{O} \\
0 \\
0\end{array}$ & 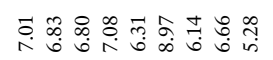 & 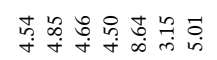 & 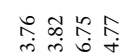 & 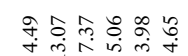 & 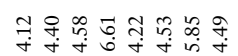 \\
\hline & 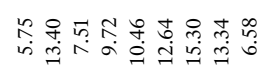 & 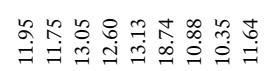 & 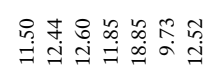 & & 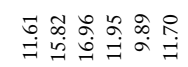 & 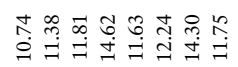 \\
\hline & 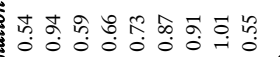 & 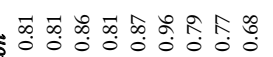 & 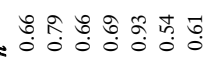 & 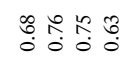 & 388 & 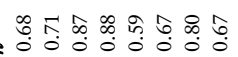 \\
\hline & & & & & & 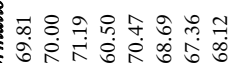 \\
\hline & & & & & & 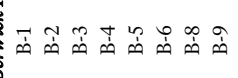 \\
\hline
\end{tabular}


while incompatible elements have lower abundance in mafic compared to felsic rocks, Cullers et al. (1997) used these ratios to determine the general characteristics of source regions of sediments. Figure 4 illustrates the range of trace element ratios for sediments derived from silicic and mafic sources (Cullers et al. 1997). La/Sc values (Fig. 4a) for the Carrabassett Formation and the Merrimack Trough formations are as low as 0.8 and show good overlap, extending to values as high as 3.6. The lower values fall within the overlap in compositions derived from mafic and silicic sources, suggesting that these formations were derived from intermediate to silicic sources. The Rangeley Formation plots within the silicic provenance range, extending to slightly higher ratios than the Carrabassett and Merrimack sediments. Likewise with Th/Sc values (Fig. $4 b$ ): all formations show some samples with intermediate compositions between mafic and silicic sources, but the Rangeley extends to slightly more silicic values. All formations have Th/Co values (Fig. 4c) in the lower range of values for silicic sources. The Cr/Th diagram (Fig. 4d) differs somewhat from the others in that the Merrimack Trough formations are dominated by intermediate values whereas the Carrabassett and Rangeley samples are silicic. On average, the Rangeley Formation has the lowest $\mathrm{Cr} / \mathrm{Th}$ values, indicating a more consistent silicic source. Even though the Kittery and Carrabassett formations have outboard paleocurrent indicators, there is no distinction in trace element ratios of these formations except that the Kittery has slightly higher $\mathrm{Cr} / \mathrm{Th}$ values (Fig. 4d) than most of the Carrabassett samples.

Chrondrite-normalized REE diagrams for Carrabassett, Rangeley, Kittery, Eliot, and Berwick formations are plotted in Fig. 5. All the analyses are quite similar and only show subtle differences in slope and in overall REE abundances. The Carrabassett Formation shows the largest range in REE abundances, with LREE between 36 and 158 times chondrite values and $(\mathrm{La} / \mathrm{Lu})_{\mathrm{N}}$ values between 2.9 and 13 (Table 2; Fig. 5a). The Rangeley Formation has the highest REE abundances and steepest slopes, with LREE ranging between 120 to 247 times chondrite values (Fig. $5 b)$ and an average $(\mathrm{La} / \mathrm{Lu})_{\mathrm{N}}$ value of 10.26. All the Merrimack Trough formations are similar in overall REE abundances and slopes. $(\mathrm{La} / \mathrm{Lu})_{\mathrm{N}}$ values are 7.0-
9.6 for the Berwick Formation, 6.8-9.9 for the Eliot, and 7.39.7 for the Kittery (Fig. 5c, 5d, and 5e). Cullers et al. (1997) found that $(\mathrm{La} / \mathrm{Lu})_{\mathrm{N}}$ values for sediments derived from silicic rocks range between 3.0 and 27 and mafic rocks between 1.1 and 7.0. The $(\mathrm{La} / \mathrm{Lu})_{\mathrm{N}}$ values for our sampled formations, regardless of provenance, lie at the lower range of silicic values, again suggesting mixed or intermediate composition source regions.

Average REE concentrations of the Rangeley and Kittery formations are shown in Fig. 5f. Because the Carrabassett Formation shows a wide range of values, average compositions for samples with LREE $>100$ and $<100$ times chondrite values are plotted. Also plotted are average values for turbidites from fore-arc, Andean-type arc and passive-margin settings (Taylor and McLennon 1985). The $>100$ times chondrite Carrabassett Formation average and the Rangeley and Kittery averages are most similar to passive margin turbidites. The $<$ 100 times chondrite Carrabassett average is more similar to fore-arc turbidites. However, none of the compatible/incompatible element ratios in the Carrabassett Formation (Fig. 4) suggest a mafic component as might be expected in a forearc setting. Additionally, all the Carrabassett, Rangeley, and Merrimack samples plot along the trailing edge turbidite field defined by McLennan et al. (1993) in the $\mathrm{Zr} / \mathrm{Sc}$ versus Th/Sc diagram (Fig. 6).

\section{$\mathrm{Nd}$ and Sr Isotopic Compositions}

Isotopic compositions of $\mathrm{Nd}$ and $\mathrm{Sr}$ for the metasedimentary rocks of the Central Maine Basin were published by Lathrop et al. (1996) and are plotted in Fig. 7 with our analyses of the Merrimack Trough formations (Table 2). Lathrop et al. (1996) included one sample of the Carrabassett Formation and two of the Littleton Formation which is thought to be the Carrabassett equivalent in New Hampshire (Moench and Pankiwskyj 1988; Hatch and Moench 1984). All samples plot below bulk-Earth values at $\varepsilon_{\mathrm{Nd}}$ values between -5 and -10 and $\mathrm{Sr}_{\mathrm{i}}$ values ranging from 0.7100 to 0.7170 . No significant isotopic difference exists between the Carrabassett/Littleton Formation and the Rangeley Formation which has Laurentian provenance. All

Table 2. Merrimack Trough $\mathrm{Nd}$ and $\mathrm{Sr}$ Isotopic Compositions.

\begin{tabular}{lcccccccc}
\hline Sample & $\mathrm{Sm}(\mathrm{ppm})$ & $\mathrm{Nd}(\mathrm{ppm})$ & ${ }^{147} \mathrm{Sm} /{ }^{144} \mathrm{Nd}$ & ${ }^{143} \mathrm{Nd} /{ }^{144} \mathrm{Nd}$ & $\mathrm{E}_{\mathrm{Nd}}(0 \mathrm{Ma})$ & $\mathrm{E}_{\mathrm{Nd}}(420 \mathrm{Ma})$ & $\mathrm{T}_{\mathrm{DM}}$ & $\mathrm{Rb}(\mathrm{ppm})$ \\
\hline B-1 & 6.30 & 31.41 & 0.1212 & 0.512058 & -11.3 & -7.3 & 1.62 & 74 \\
B-3 & 7.96 & 40.88 & 0.1177 & 0.512015 & -12.2 & -7.9 & 1.63 & 94 \\
B-6 & 6.63 & 31.83 & 0.1260 & 0.512008 & -12.3 & -8.5 & 1.80 & 89 \\
B-9 & 6.07 & 30.12 & 0.1218 & 0.512069 & -11.1 & -7.1 & 1.61 & 82 \\
E-1 & 6.36 & 32.58 & 0.1181 & 0.511977 & -12.9 & -8.7 & 1.70 & 74 \\
E-3 & 5.07 & 25.85 & 0.1185 & 0.511999 & -12.5 & -8.3 & 1.67 & 130 \\
K-6 & 7.26 & 37.29 & 0.1177 & 0.511981 & -12.8 & -8.6 & 1.68 & 168 \\
K-9 & 6.16 & 30.49 & 0.1221 & 0.511969 & -13.1 & -9.1 & 1.79 & 117 \\
K-11 & 5.47 & 27.03 & 0.1224 & 0.512000 & -12.4 & -8.5 & 1.74 & 65 \\
\hline
\end{tabular}

DorAIS ET AL - Insights into the Acadian Orogeny, New England Appalachians ... 

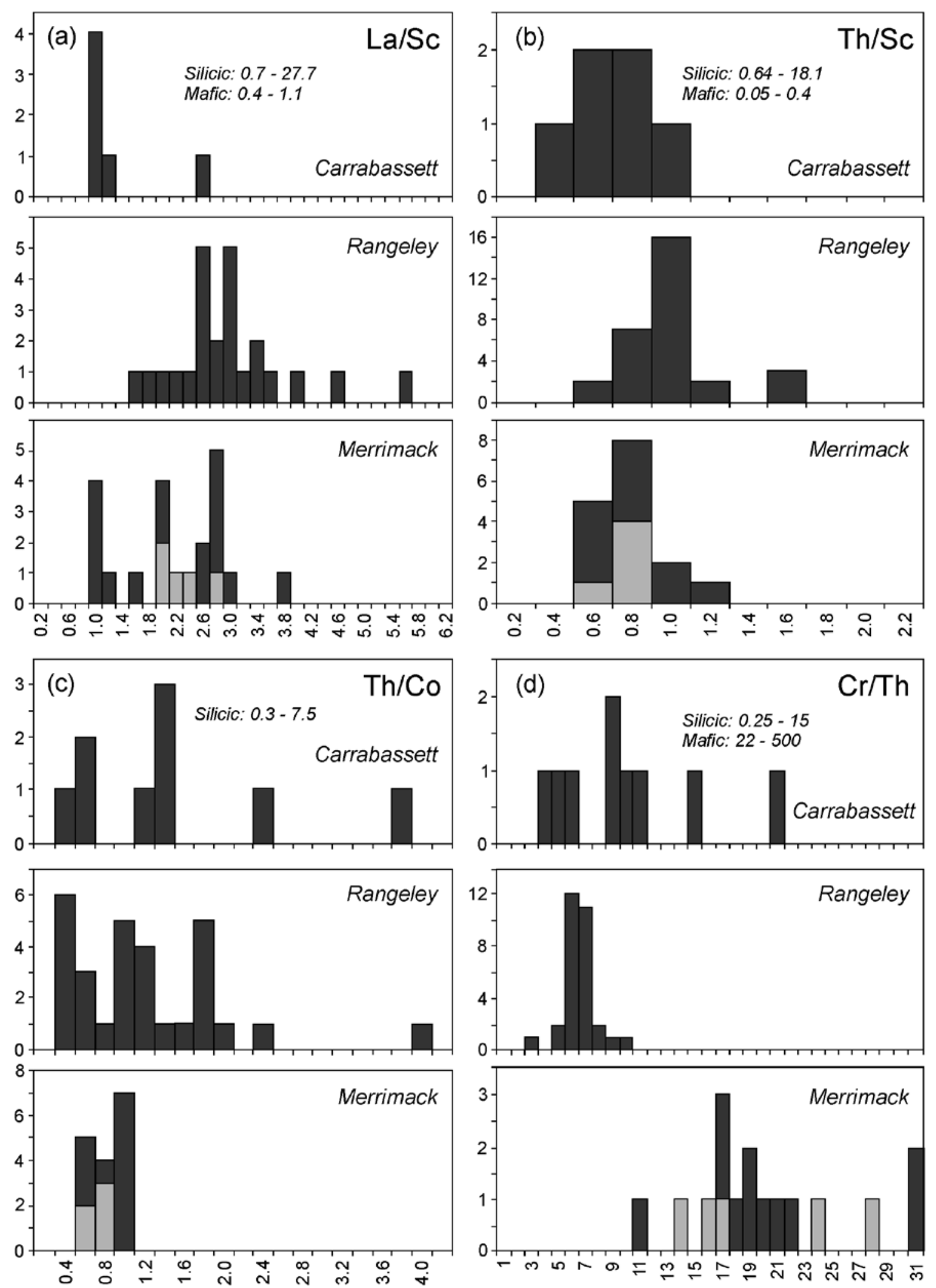

Fig. 4. Histograms of incompatible/compatible element ratios (La/Ca, Fig 4a; Th/Sc, Fig. 4b; Th/Co, Fig. 4c) and compatible/incompatible element ratio (Cr/Th, Fig. 4d). In each histogram, the Carrabassett, Rangeley, and Merrimack Trough formations (Kittery Formation shown in light grey) show similar ratios, plotting at the more mafic end of the silicic range of ratios, suggesting intermediate compositions for the source regions. Silicic and mafic source rock ratios are after Cullers et al. (1997). 

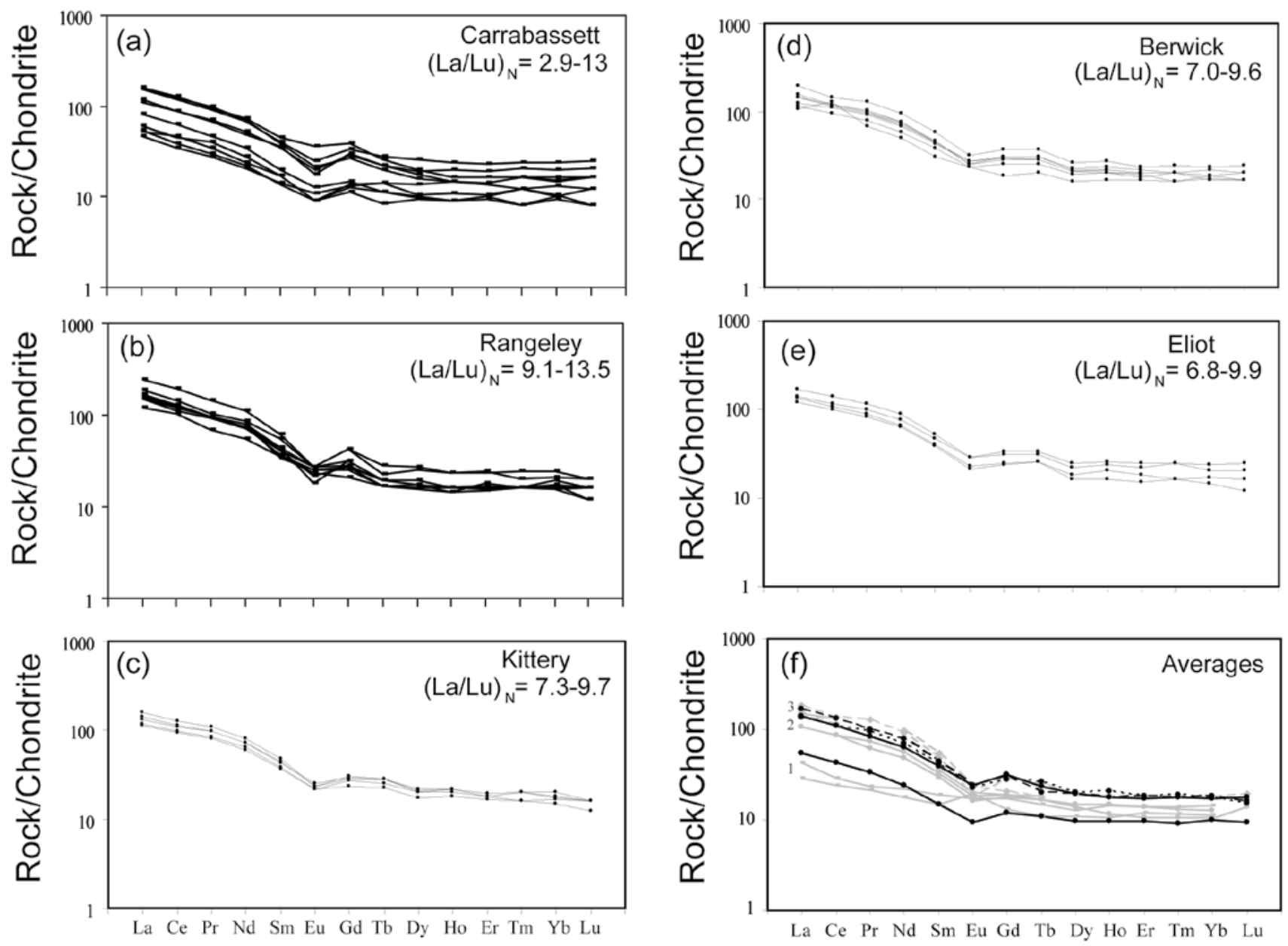

Fig. 5. Chondrite-normalized REE patterns for the Carrabassett, Rangeley and Merrimack Trough formations and averages for each formation. The Carrabassett Formation shows a range of REE enrichment, but the samples with LREE values 100 times chondrite values are similar to those of the Rangeley and Merrimack Trough formations. Also plotted are REE patterns of average turbidites from fore-arc (1), Andean margin (2), and passive margin (3) settings (Fig. 5c with averages from Taylor and McLennan 1985). The $>100$ times Carrabassett average (solid line) and the Rangeley (dashed line) and Kittery (dotted line) averages are most similar to passive margin turbidites. The $<100$ times chondritic Carrabassett Formation samples (solid line) are similar to fore-arc turbidites.

Merrimack Trough samples, including those from the Kittery Formation, plot in the Central Maine Basin field, identical to the Carrabassett and Rangeley formation samples. Thus, regardless of whether the Carrabassett and Kittery formations have paleocurrent indicators of an offshore source, their isotopic signatures are no different from the Rangeley Formation metasedimentary rocks that were derived from Laurentia.

Carrabassett/Littleton Formation Nd model ages average 1.7 Ma (Lathrop et al. 1996) as do the averages of the formations from the Merrimack Trough (Table 2). Rangeley Formation and other Laurentia-derived formations have Nd model ages that average $1.61 \mathrm{Ma}$ (Lathrop et al. 1996). These ages are similar to the non-altered samples of Cullers $\mathrm{et} \mathrm{al}$. (1997) and Bock et al. (2004), suggesting that the samples of Lathrop et al. (1996) represent original provenance signatures.

McLennan et al. (1993) combined Nd isotopic data with Th/
Sc ratios to distinguish active arc and trailing edge turbidites (Fig. 8). Active arc turbidites define the non-quartzose field, comprising sediments that are dominated by mafic sources. The majority of these sediments plot at positive $\varepsilon_{\mathrm{Nd}}$ and low $\mathrm{Th} / \mathrm{Sc}$ values. In contrast, turbidites derived from trailing edge settings are quartzose with negative $\varepsilon_{\mathrm{Nd}}$ values. The Merrimack Trough formations plot in the quartzose field with no distinction between the Kittery Formation and the Eliot/Berwick analyses. Although we lack Nd isotopic data on our Carrabassett Formation samples, they have the same $\mathrm{Th} / \mathrm{Sc}$ values as the Merrimack metasedimentary rocks and Lathrop et al. (1996) reported the same range in $\varepsilon_{\mathrm{Nd}}$ values for the Littleton as the Merrimack; thus the Carrabassett Formation would plot among the Merrimack samples which are derived from a nonjuvenile, quartzose source. 


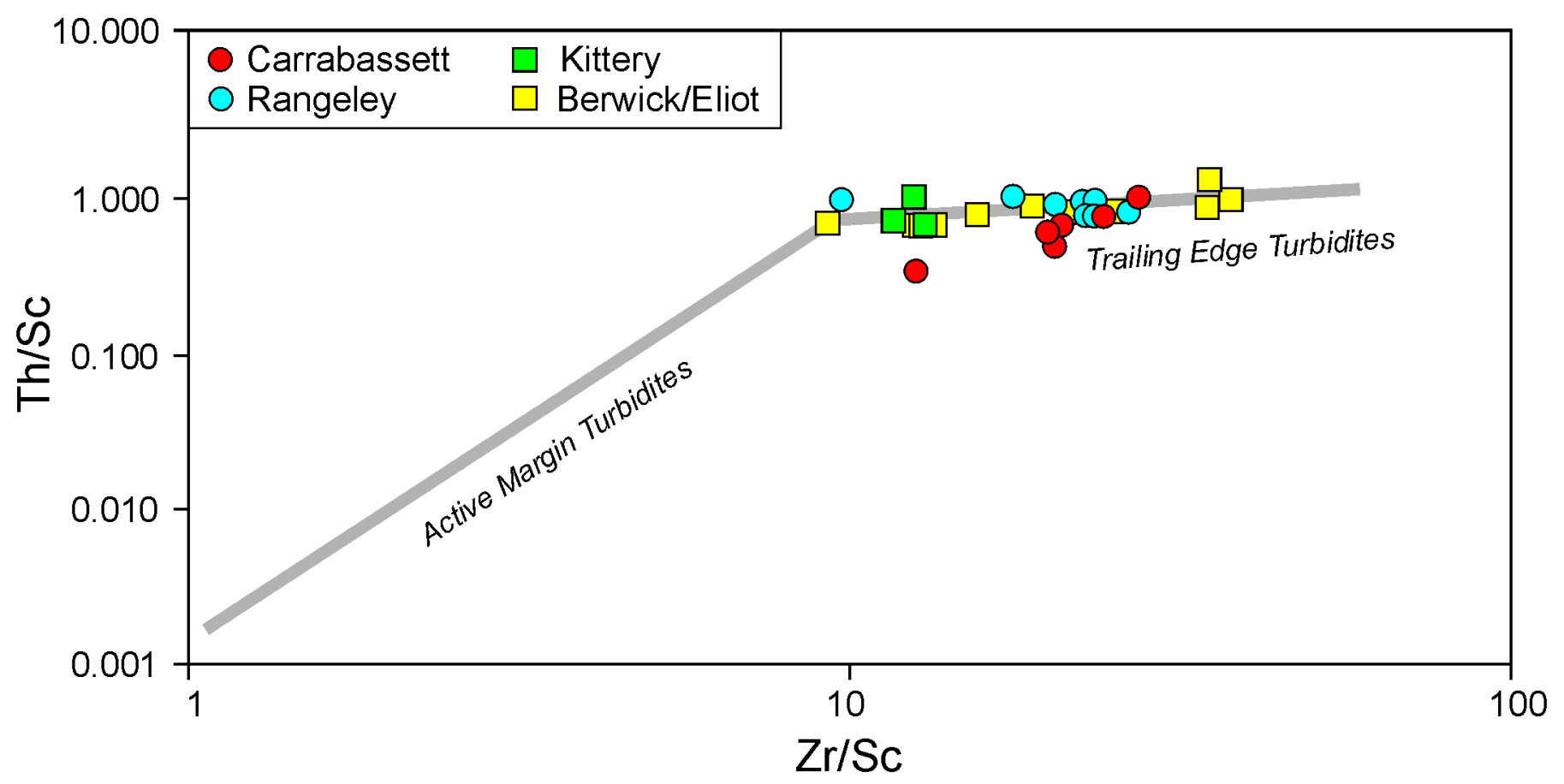

Fig. 6. Zr/Sc versus Th/Sc diagram (after McLennan et al. 1993). Carrabassett, Rangeley, Kittery and Berwick/Eliot formations all plot as trailing edge turbidites.

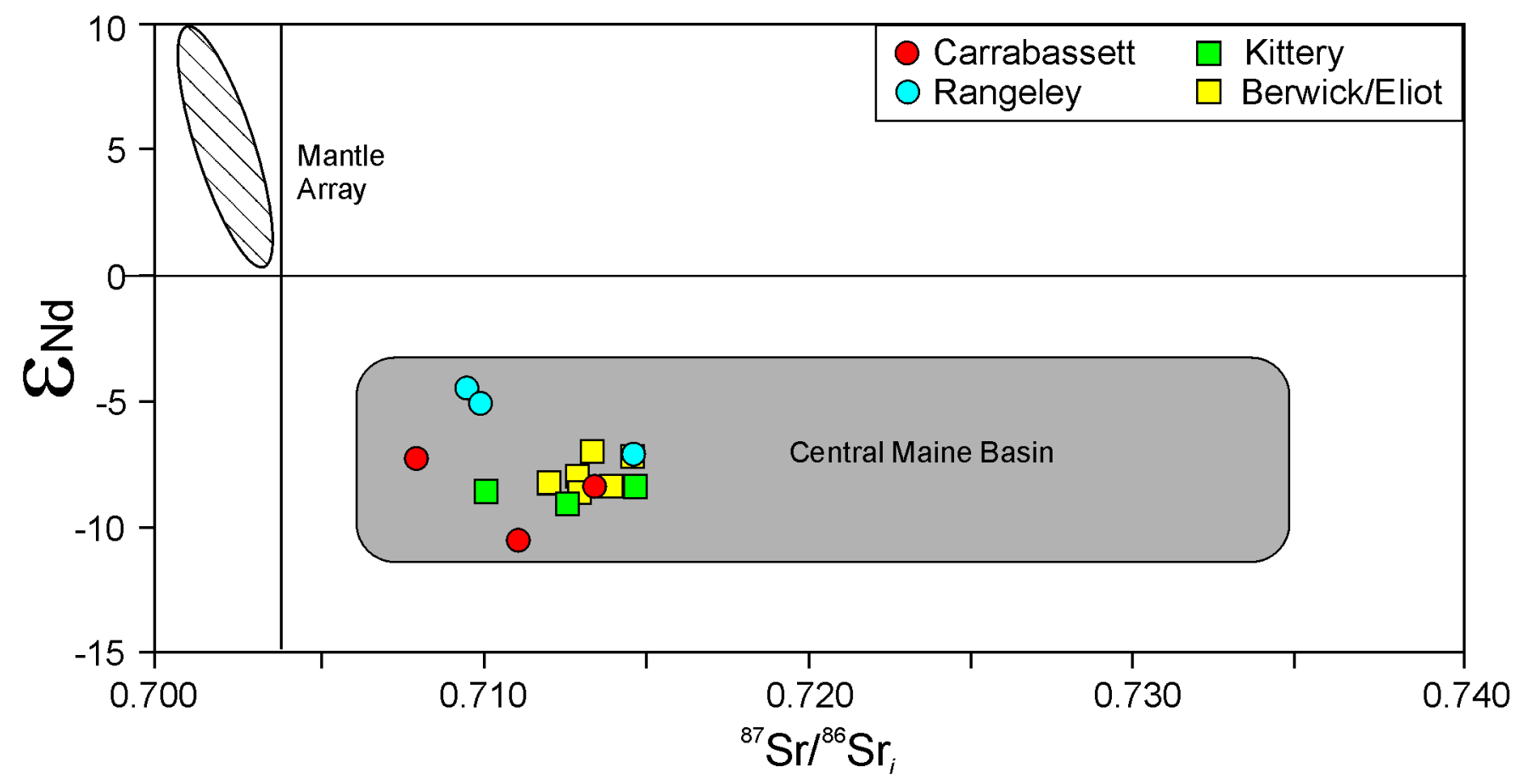

Fig. 7. Initial Sr versus $\varepsilon_{\mathrm{Nd}}(420 \mathrm{Ma})$ values showing data from the Carrabassett/Littleton Formation and other Central Maine Basin metasedimentary rocks (grey field; data from Lathrop et al. 1996). Although paleocurrent data indicate provenance from the east, the Carrabassett Formation has isotopic values identical to Laurentia-derived sediments, suggesting an older mature crustal component in the Carrabassett Formation. Similarly, the Kittery and Berwick/Eliot formations have identical bulk-rock isotopic values. 


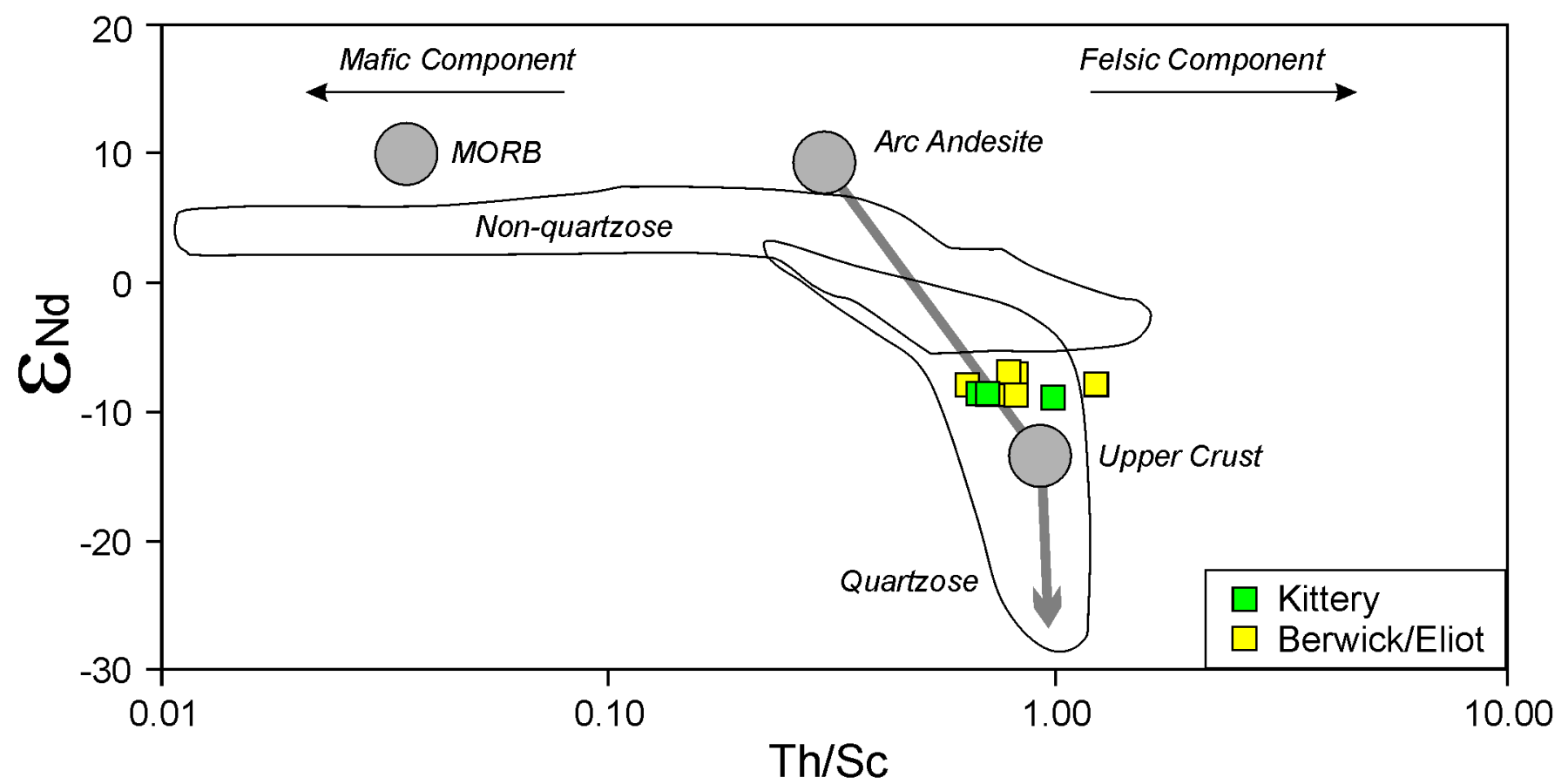

Fig. 8. Th/Sc versus $\varepsilon_{\mathrm{Nd}}$ diagram (after McLennan et al. 1993), with $\varepsilon_{\mathrm{Nd}}$ calculated at $413 \mathrm{Ma}$. The Berwick/Eliot and Kittery formation samples plot in the quartzose, upper crustal provenance field. Although no isotopic data for our Carrabassett Formation samples are available, our samples have the same $\mathrm{Th} / \mathrm{Sc}$ ratios, and the Carrabassett samples of Lathrop et al. (1996) have the same $\varepsilon_{\mathrm{Nd}}$ values, as the Berwick/Eliot and Kittery samples.

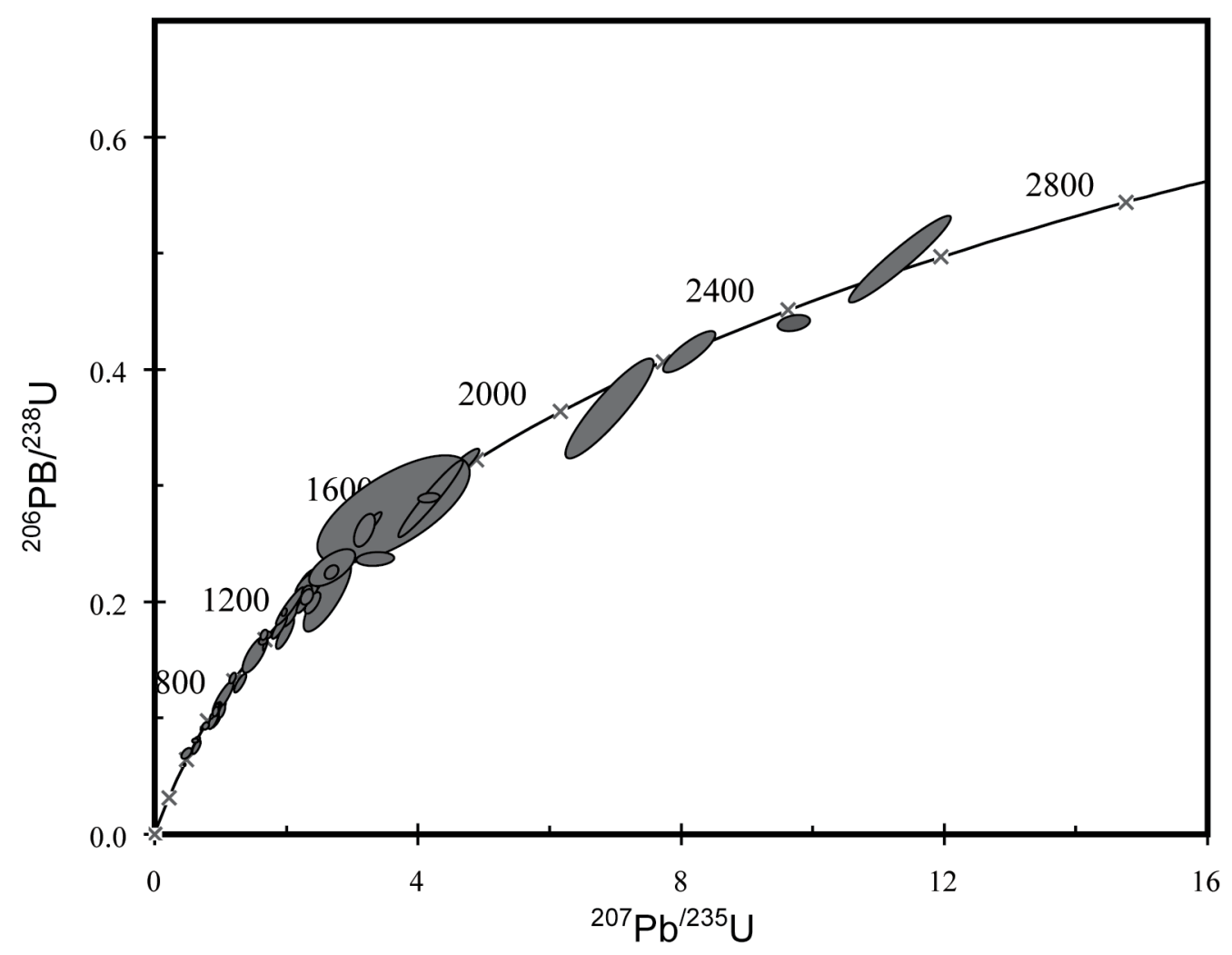

Fig. 9. ${ }^{207} \mathrm{~Pb} /{ }^{235} \mathrm{U}$ versus ${ }^{206} \mathrm{~Pb} /{ }^{238} \mathrm{U}$ concordia diagram for the Carrabassett Formation. 


\section{Detrital Zircon Geochronology}

A plot of ${ }^{207} \mathrm{~Pb} /{ }^{235} \mathrm{U}$ versus ${ }^{206} \mathrm{~Pb} /{ }^{238} \mathrm{U}$ for detrital zircons from the Carrabassett Formation is shown in Fig. 9. Plotted data are within $\pm 10 \%$ of concordancy and the data point error ellipses are $1 \sigma$. The complete data set is given in Table 3 . A relative probability and histogram diagram is plotted in Fig. 10a. A potential Acadian zircon fraction is present with an age of $412 \pm 23 \mathrm{Ma}$. Zircon grains with Taconic-like ages are present also, with ages of $445 \pm 29$ and $482 \pm 12 \mathrm{Ma}$. A dominant peak is centered at $\sim 600 \mathrm{Ma}$. The most prominent peak on the diagram ranges from 900 to $1440 \mathrm{Ma}$, centered at $1200 \mathrm{Ma}$. Individual grains have older ages of $1500-1600,1800,2080$, 2200 , and $2600 \mathrm{Ma}$. The paucity of $\sim 600 \mathrm{Ma}$ and $1490-1610$ zircons in Laurentia presents an obvious difference between
Laurentia and the Carrabassett Formation (Sampson et al. 2005). Likewise, two grains centered around $2280 \mathrm{Ma}$ in the Carrabassett Formation contrast with the absence of grains of that age in Avalonia (Samson et al. 2005).

Comparisons of Carrabassett Formation detrital zircons with available zircon ages from Ganderia are presented in Fig. 10b. Ganderia ages are for inherited zircons derived from plutonic rocks and boulders thought to represent the unexposed basement (Roddick and Bevier 1995; van Staal et al. 1996), from orthogneisses of the Lyme Dome, Connecticut (Walsh et al. 2007), and from metasedimentary rocks east of the Dog Bay Line, Newfoundland (Pollock et al. 2007). Good matches are evident between the Carrabassett and Ganderia supracrustal rocks with strong similarities at $\sim 600 \mathrm{Ma}$ and the common peak centered at $\sim 1200 \mathrm{Ma}$.

Table 3. LA-ICPMS Detrital Zircon Data for the Carrabassett Formation, Maine.

\begin{tabular}{|c|c|c|c|c|c|c|c|c|c|c|c|c|c|c|}
\hline Analyses & ${ }^{207} \mathrm{~Pb} /{ }^{235} \mathrm{U}$ & $\pm 1 \sigma$ & ${ }^{206} \mathrm{~Pb} /{ }^{238} \mathrm{U}$ & $\pm 1 \sigma$ & Rho & $\begin{array}{c}{ }^{207} \mathrm{~Pb} /{ }^{235} \mathrm{U} \\
(\mathrm{Ma})\end{array}$ & $\pm 1 \sigma$ & $\begin{array}{c}{ }^{206} \mathrm{~Pb} /{ }^{238} \mathrm{U} \\
(\mathrm{Ma})\end{array}$ & $\pm 1 \sigma$ & ${ }^{207} \mathrm{~Pb} /{ }^{206} \mathrm{~Pb}$ & $\pm 1 \sigma$ & $\begin{array}{c}{ }^{207} \mathrm{~Pb} /{ }^{206} \mathrm{~Pb} \\
(\mathrm{Ma})\end{array}$ & $\pm 1 \sigma$ & $\begin{array}{c}\% \\
\text { conc. }\end{array}$ \\
\hline de07A08 & 0.8252 & 0.0178 & 0.0977 & 0.0016 & 0.38 & 610.9 & 9.9 & 601.1 & 9.3 & 0.0614 & 0.0013 & 654.9 & 45.6 & 92 \\
\hline de07A09 & 1.6447 & 0.0319 & 0.1604 & 0.0046 & 0.73 & 987.5 & 12.3 & 959.1 & 25.4 & 0.0733 & 0.0018 & 1021.2 & 45.9 & 94 \\
\hline de07A15 & 3.3177 & 0.2401 & 0.2340 & 0.0051 & 0.15 & 1485.2 & 56.5 & 1355.3 & 26.5 & 0.1041 & 0.0061 & 1498.9 & 107.6 & 90 \\
\hline de07A20 & 1.9294 & 0.0333 & 0.1882 & 0.0027 & 0.42 & 1091.3 & 11.5 & 1111.7 & 14.8 & 0.0767 & 0.0011 & 1113.3 & 29.3 & 100 \\
\hline de07A23 & 1.6611 & 0.0519 & 0.1685 & 0.0026 & 0.24 & 993.8 & 19.8 & 1003.6 & 14.1 & 0.0732 & 0.0022 & 1020.8 & 61.0 & 98 \\
\hline de07A24 & 2.2732 & 0.0631 & 0.2066 & 0.0040 & 0.35 & 1204.0 & 19.6 & 1210.6 & 21.2 & 0.0825 & 0.0013 & 1257.5 & 31.6 & 96 \\
\hline de07A27 & 4.1297 & 0.1255 & 0.2870 & 0.0037 & 0.21 & 1660.2 & 24.8 & 1626.5 & 18.4 & 0.1069 & 0.0025 & 1646.9 & 42.7 & 99 \\
\hline de07A28 & 0.9483 & 0.0157 & 0.1082 & 0.0012 & 0.33 & 677.2 & 8.2 & 662.6 & 7.0 & 0.0663 & 0.0009 & 714.5 & 29.3 & 93 \\
\hline de07A33 & 2.2759 & 0.0832 & 0.2010 & 0.0053 & 0.36 & 1204.8 & 25.8 & 1180.8 & 28.6 & 0.0846 & 0.0032 & 1307.3 & 73.9 & 90 \\
\hline de07A34 & 0.7311 & 0.0387 & 0.0896 & 0.0026 & 0.27 & 557.2 & 22.7 & 552.9 & 15.4 & 0.0626 & 0.0017 & 595.8 & 58.8 & 93 \\
\hline de07A35 & 0.8828 & 0.0414 & 0.1018 & 0.0035 & 0.36 & 642.5 & 22.3 & 625.1 & 20.2 & 0.0623 & 0.0031 & 683.0 & 104.5 & 92 \\
\hline de07A51 & 3.1574 & 0.1283 & 0.2591 & 0.0115 & 0.55 & 1446.8 & 31.3 & 1485.3 & 58.8 & 0.0906 & 0.0052 & 1438.0 & 110.1 & 103 \\
\hline de07A52 & 0.6643 & 0.0143 & 0.0872 & 0.0012 & 0.33 & 517.2 & 8.7 & 538.8 & 7.2 & 0.0570 & 0.0012 & 591.1 & 45.4 & 91 \\
\hline de07A57 & 2.6483 & 0.0849 & 0.2226 & 0.0049 & 0.34 & 1314.2 & 23.6 & 1295.4 & 25.8 & 0.0893 & 0.0025 & 1409.8 & 52.9 & 92 \\
\hline de07A58 & 1.5951 & 0.0505 & 0.1629 & 0.0025 & 0.24 & 968.3 & 19.8 & 972.9 & 13.8 & 0.0739 & 0.0021 & 1039.6 & 57.4 & 94 \\
\hline de07A59 & 9.6986 & 0.2020 & 0.4384 & 0.0056 & 0.31 & 2406.6 & 19.2 & 2343.4 & 25.1 & 0.1659 & 0.0019 & 2516.2 & 19.5 & 93 \\
\hline de07A61 & 1.6287 & 0.0482 & 0.1687 & 0.0034 & 0.34 & 981.4 & 18.6 & 1005.0 & 18.6 & 0.0723 & 0.0026 & 994.0 & 72.6 & 101 \\
\hline oc02a08 & 2.6505 & 0.1441 & 0.2165 & 0.0126 & 0.7947 & 1314.8 & 40.1 & 1263.5 & 66.9 & 0.0814 & 0.0012 & 1230.3 & 29.6 & 103 \\
\hline oc02a09 & 2.2853 & 0.1415 & 0.2130 & 0.0107 & 0.6878 & 1207.7 & 43.7 & 1244.7 & 57.0 & 0.0775 & 0.0015 & 1135.2 & 39.5 & 110 \\
\hline oc02a13 & 1.9623 & 0.1002 & 0.1871 & 0.0084 & 0.7302 & 1102.7 & 34.3 & 1105.7 & 45.4 & 0.0749 & 0.0011 & 1066.5 & 30.4 & 104 \\
\hline oc02a16 & 3.3807 & 0.2466 & 0.2656 & 0.0119 & 0.7048 & 1499.9 & 57.2 & 1518.6 & 60.6 & 0.0950 & 0.0014 & 1527.7 & 27.7 & 99 \\
\hline oc02a17 & 1.9400 & 0.1105 & 0.1702 & 0.0111 & 0.8114 & 1095.0 & 38.2 & 1013.1 & 61.3 & 0.0746 & 0.0014 & 1056.9 & 37.6 & 96 \\
\hline oc02a18 & 2.3298 & 0.1854 & 0.2114 & 0.0102 & 0.5965 & 1221.4 & 56.5 & 1236.1 & 54.4 & 0.0827 & 0.0019 & 1262.4 & 44.8 & 98 \\
\hline oc02a19 & 2.4207 & 0.1340 & 0.2116 & 0.0118 & 0.8149 & 1248.8 & 39.8 & 1237.4 & 62.9 & 0.0787 & 0.0011 & 1165.4 & 28.5 & 106 \\
\hline oc02a20 & 2.0020 & 0.1027 & 0.1862 & 0.0087 & 0.6576 & 1116.2 & 34.7 & 1100.9 & 47.0 & 0.0741 & 0.0015 & 1044.7 & 41.8 & 105 \\
\hline oc02a24 & 2.5732 & 0.1123 & 0.2149 & 0.0079 & 0.7052 & 1293.1 & 31.9 & 1254.9 & 41.7 & 0.0828 & 0.0014 & 1263.4 & 32.4 & 99 \\
\hline oc02a 25 & 6.8820 & 0.5480 & 0.3642 & 0.0350 & 0.9065 & 2096.3 & 70.6 & 2002.2 & 165.2 & 0.1298 & 0.0013 & 2094.8 & 18.0 & 96 \\
\hline oc02a32 & 0.4436 & 0.0608 & 0.0660 & 0.0039 & 0.5269 & 372.8 & 42.8 & 412.2 & 23.9 & 0.0545 & 0.0016 & 390.4 & 65.8 & 106 \\
\hline oc02a33 & 0.5877 & 0.0489 & 0.0715 & 0.0048 & 0.7225 & 469.4 & 31.3 & 445.3 & 28.8 & 0.0558 & 0.0011 & 445.8 & 42.6 & 100 \\
\hline oc02a34 & 0.9561 & 0.0655 & 0.1043 & 0.0062 & 0.7499 & 681.3 & 34.0 & 639.8 & 36.4 & 0.0621 & 0.0011 & 678.7 & 38.1 & 94 \\
\hline oc02a37 & 0.9984 & 0.1332 & 0.1143 & 0.0118 & 0.8701 & 703.0 & 67.7 & 697.6 & 68.3 & 0.0621 & 0.0010 & 677.4 & 33.3 & 103 \\
\hline oc02a39 & 0.8848 & 0.0621 & 0.0961 & 0.0061 & 0.674 & 643.6 & 33.5 & 591.2 & 36.0 & 0.0603 & 0.0014 & 615.6 & 49.9 & 96 \\
\hline oc02a46 & 0.8416 & 0.0651 & 0.0950 & 0.0062 & 0.7173 & 620.0 & 35.9 & 584.8 & 36.6 & 0.0581 & 0.0013 & 533.1 & 48.8 & 110 \\
\hline oc02a47 & 2.5768 & 0.2926 & 0.2017 & 0.0247 & 0.8104 & 1294.1 & 83.1 & 1184.7 & 132.6 & 0.0853 & 0.0020 & 1322.2 & 45.8 & 90 \\
\hline oc02a48 & 1.4787 & 0.1459 & 0.1513 & 0.0128 & 0.7572 & 921.7 & 59.8 & 908.2 & 71.7 & 0.0671 & 0.0015 & 840.4 & 48.1 & 108 \\
\hline oc02a54 & 2.3666 & 0.0973 & 0.1963 & 0.0078 & 0.6711 & 1232.6 & 29.3 & 1155.3 & 41.8 & 0.0810 & 0.0013 & 1221.6 & 31.4 & 95 \\
\hline oc02a62 & 8.0972 & 0.3297 & 0.4137 & 0.0145 & 0.8406 & 2241.9 & 36.8 & 2231.6 & 66.3 & 0.1390 & 0.0012 & 2214.8 & 15.3 & 101 \\
\hline oc02b03 & 4.6092 & 0.2345 & 0.3143 & 0.0125 & 0.9084 & 1750.9 & 42.5 & 1761.6 & 61.3 & 0.1079 & 0.0008 & 1764.9 & 13.2 & 100 \\
\hline oc02b04 & 3.5973 & 0.9486 & 0.2772 & 0.0381 & 0.6823 & 1548.9 & 209.5 & 1577.2 & 192.5 & 0.1044 & 0.0020 & 1704.4 & 35.9 & 93 \\
\hline oc02b06 & 2.6590 & 0.2839 & 0.2268 & 0.0132 & 0.6439 & 1317.2 & 78.8 & 1317.6 & 69.3 & 0.0874 & 0.0015 & 1369.5 & 32.6 & 96 \\
\hline oc02b12 & 2.2238 & 0.0936 & 0.1959 & 0.0072 & 0.8044 & 1188.6 & 29.5 & 1153.3 & 39.0 & 0.0799 & 0.0008 & 1194.1 & 19.9 & 97 \\
\hline oc02b14 & 4.1626 & 0.4077 & 0.2866 & 0.0271 & 0.963 & 1666.7 & 80.2 & 1624.4 & 135.5 & 0.1035 & 0.0007 & 1688.7 & 13.3 & 96 \\
\hline oc03a05 & 0.9035 & 0.0408 & 0.1058 & 0.0034 & 0.7269 & 653.6 & 21.8 & 648.4 & 19.6 & 0.0629 & 0.0007 & 703.3 & 23.0 & 92 \\
\hline oc03a07 & 1.8559 & 0.0943 & 0.1749 & 0.0079 & 0.8471 & 1065.5 & 33.5 & 1039.3 & 43.2 & 0.0765 & 0.0008 & 1106.9 & 20.5 & 94 \\
\hline oc03a08 & 3.2204 & 0.1598 & 0.2627 & 0.0103 & 0.916 & 1462.1 & 38.5 & 1503.9 & 52.4 & 0.0927 & 0.0006 & 1481.5 & 12.3 & 102 \\
\hline oc03a11 & 11.3083 & 0.6306 & 0.4939 & 0.0307 & 0.9545 & 2548.9 & 52.0 & 2587.4 & 132.7 & 0.1671 & 0.0009 & 2528.5 & 9.0 & 102 \\
\hline oc03b04 & 0.6002 & 0.0289 & 0.0777 & 0.0020 & 0.4382 & 477.4 & 18.3 & 482.6 & 12.0 & 0.0561 & 0.0010 & 458.1 & 39.2 & 105 \\
\hline oc03b05 & 1.1466 & 0.0504 & 0.1312 & 0.0037 & 0.5195 & 775.6 & 23.9 & 794.8 & 21.2 & 0.0648 & 0.0012 & 766.5 & 39.5 & 104 \\
\hline oc03b08 & 2.0178 & 0.1708 & 0.1936 & 0.0135 & 0.9342 & 1121.5 & 57.5 & 1141.1 & 72.9 & 0.0798 & 0.0007 & 1191.1 & 18.4 & 96 \\
\hline
\end{tabular}




\section{DISCUSSION}

\section{Bulk-Rock Geochemistry}

The above results show that there are no significant bulkrock geochemical differences between Laurentia-derived sediments as typified by our Rangeley Formation samples and the easterly derived Carrabassett and Kittery formations. The Carrabassett Formation, with an outboard source, has similar trace element ratios as the Rangeley Formation. Likewise, the Kittery Formation, which also has outboard paleocurrent indicators, is no different than the Berwick and Eliot formations of the Merrimack Trough nor is it significantly different from the Laurentia-derived Rangeley Formation. Thus all the formations appear to have been derived from source regions that were neither dominated by mafic rocks such as found in primitive arcs nor exclusively by silicic rocks. Rather, it is plausible that the sediments were produced from large areas that homogenized to an average intermediate composition. The in- termediate composition is also supported by REE abundances. The Rangeley, Berwick, Eliot, and Kittery formations and the $>100$ times chondritic portion of the Carrabassett Formation all have REE abundances most similar to passive margin turbidites (Fig. 5), an inference supported by the $\mathrm{Zr} / \mathrm{Sc}$ verus Th/ Sc diagram (Fig. 6).

Isotopic data (Fig. 7) show no differences between Central Maine Basin metasedimentary rocks, including the Rangeley Formation, and either the Carrabassett Formation or the Merrimack Trough formations. All the metasedimentary samples fall in a relatively narrow range of $\varepsilon_{\mathrm{Nd}}$ values between - 6 and -10 . This range differs considerably from Avalonian granites commonly used to define the basement of Avalonia (Nance and Murphy 1994; Nance et al. 2002; Murphy and Nance 2002) in that most of the granites have positive $\varepsilon_{\mathrm{Nd}}$ values. Many workers have therefore concluded that Avalonia consists primarily of juvenile crust which is isotopically distinct from older materials derived from Laurentia. In contrast, Ganderian plutons tend to have negative $\varepsilon_{\mathrm{Nd}}$ values of the same range as the
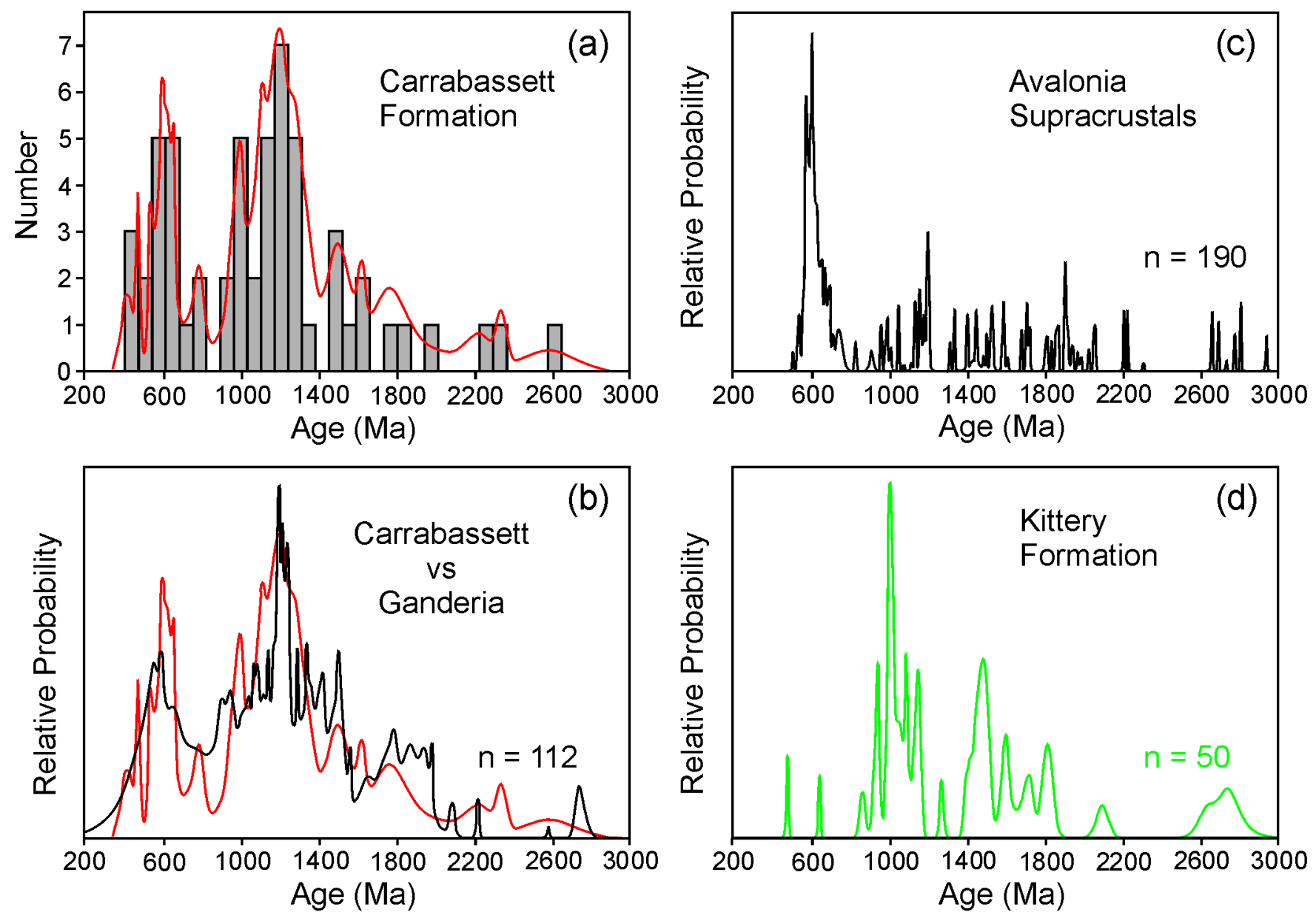

Fig. 10. (a) Histogram and relative probability plot of zircon ages, Carrabassett Formation. (b) Comparison of Carrabassett Formation (red) and Ganderian (black) relative probability plots. Ganderian ages are from Roddick and Bevier (1995), van Staal et al. (1996), Walsh et al. (2007), and Pollock et al. (2007). (c) Relative probability plot of zircon ages from Avalonian metasedimentary rocks (Keppie et al. 1998; Barr et al. 2003; Murphy et al. 2004). (d) Relative probability plot of zircon ages from the Kittery Formation (Wintsch et al. 2007). 
Carrabassett and Kittery metasedimentary rocks (Roddick and Bevier 1995; van Staal et al. 1996). The Nd model ages of the Carrabassett Formation (Lathrop et al. 1996) and the Kittery Formation average $1.70 \mathrm{Ma}$ and $1.74 \mathrm{Ma}$, respectively. These ages are far older than the age of Avalonia, which is dominated by juvenile arc rocks of $\sim 600 \mathrm{Ma}$ and Neoproterozoic model ages (Nance and Murphy 1994; Nance et al. 2002; Murphy and Nance 2002), but are similar to Ganderian model ages (Whalen et al. 1996, 1997; Schofield and D'Lemos 2000). Thus it appears that both the Carrabassett and Kittery formations potentially match Ganderian but not Avalonian basement.

The picture is complicated when one considers the Neoproterozoic through Cambrian supracrustal rocks associated with Avalonia. A recent study by Satkoski et al. (2007) showed that some of the sedimentary cover rocks of the Avalonian Caledonia terrane of New Brunswick have positive $\varepsilon_{\mathrm{Nd}}$ values and are interpreted as being derived from Avalonian sources. However, other metasedimentary units have negative $\varepsilon_{\mathrm{Nd}}$ values and were interpreted as originating from a large, isotopically mature source (Samson et al. 2000; Murphy 2002; Satkoski et al. 2007). These Avalonian supracrustal rocks are interpreted as being derived from older, Gondwanan sources and deposited on Avalonian basement (Satkoski et al. 2007). Therefore, based on bulk-rock $\varepsilon_{\mathrm{Nd}}$ values alone, we cannot exclude the possibility that the Carrabassett Formation could be dominated by recycled Avalonian supracrustal rocks rather than juvenile Avalonian basement. However, the abundance of Avalonian supracrustal rocks with mature crustal signatures appears to be relatively small and the odds of the Carrabassett Formation exclusively representing recycling of such supracrustal rocks seems low. Additionally, the available detrital zircon ages of these supracrustal rocks are considerably different than those of the Carrabassett Formation (compare Fig. 10a with 10c).

It appears that no bulk-rock characteristics, either trace element or isotopic, can clearly distinguish the Laurentia-derived Rangeley from peri-Gondwanan Carrabassett or Kittery formations. As it has been shown that Quaternary sediments sampling large continental masses have remarkably uniform $\varepsilon_{\mathrm{Nd}}$ values of $\sim-11$ and $\mathrm{T}_{\mathrm{DM}}$ of $1.9 \mathrm{Ga}$ (Goldstein et al. 1984, 1997; Goldstein and Jacobsen 1988), we interpret this lack of unique bulk-rock compositions of sediments derived from Laurentia (Rangeley Formation) and Gondwana (Carrabassett/Kittery formations) as signifying that the formations represent sediments derived from large regions of mature continental crust which homogenized their bulk-rock characteristics. These characteristics differ from the juvenile Avalonian crust, but not necessarily from Avalonian supracrustal rocks.

\section{Geochronology}

Although there are similarities between the ages of detrital zircon grains in the Carrabassett Formation and those of Laurentia, a noticeable difference is the paucity of $\sim 600$ and 1490 to 1610 Ma zircons in the Laurentian populations, which points to a non-Laurentian component for the Carrabassett Formation sedimentary rocks. The Carrabassett Formation ages cluster at $\sim 1200 \mathrm{Ma}$, perhaps representing a Grenvillian component derived from Laurentia, but as demonstrated by van Staal et al. (1996), such ages are also common in periGondwanan Ganderia. Thus, based on detrital zircon geochronology, a Ganderian source is plausible. Figure 10b compares relative probability plots for Carrabassett Formation zircons with those of Ganderia (Roddick and Bevier 1995; van Staal et al. 1996; Pollock et al. 2007; Walsh et al. 2007). There are strong similarities at 500-700 Ma, 1000-1400 Ma, and 1750 $\mathrm{Ma}$. These similarities, along with the similar Nd isotopic values and model ages, and a passive margin depositional setting for Ganderia (Williams 1979; van der Pluijm and van Staal 1988) that is also characteristic of the Carrabassett Formation (Fig. $5 \mathrm{f}$ and Fig. 6), indicate that Ganderia may have been the source for the easterly derived sediments that became the Carrabassett Formation.

In contrast, the detrital zircon ages from Avalonian metasedimentary rocks (Keppie et al. 1998; Barr et al. 2003; Murphy et al. 2004) shown in Fig. 10c have some similarities to the Carrabassett Formation ages, but are of vastly different proportions. The Kittery Formation of the Merrimack Trough has a relative probability plot that is also different from the Carrabassett Formation, even though Wintsch et al. (2007) considered the Kittery Formation to be derived from Gondwana. The Kittery Formation has a prominent peak at $\sim 1000 \mathrm{Ma}$ whereas the most prominent peak in the Carrabassett Formation is at $\sim 1200 \mathrm{Ma}$. Additionally, the Carrabassett Formation has a well-defined peak at $~ 600 \mathrm{Ma}$; the Kittery Formation yielded only one zircon of that age. Therefore, even though the Kittery Formation has an outboard source that may be Gondwanan (Wintsch et al. 2007), the detritial zircon ages indicate that this source was different from that of the Carrabassett Formation. This difference may not be a surprise given the up to $70 \mathrm{Ma}$ age difference between the two formations.

\section{Provenance and tectonic setting of the Carrabassett Formation}

The criteria for assigning Ganderian provenance to the Carrabassett Formation are summarized in Fig. 11. Although some bulk-rock characteristics match other terranes, one or more mismatches suggest that these terranes were not Carrabassett Formation sources. Paleocurrent indicators suggest an outboard source, suggestive of either Ganderia or Avalonia. Bulk-rock major and trace element concentrations, the bulk-rock $\mathrm{Nd}$ isotopic compositions, and the $\mathrm{Nd}$ model ages do not discriminate between Ganderian, Avalonian supracrustal, or Laurentian sources because all three of those sources represent mature, continental crust. These parameters do however, eliminate the juvenile arc portion of Avalonia, and since this is the dominant component of Avalonia, these mismatches minimize the possibility of an Avalonian provenance. The detrital zircon ages eliminate Laurentia and the younger juvenile Avalonia as sources. While the Avalonian supracrustal rocks 
are permissible source rocks for the Carrabassett sediments based on bulk-rock characteristics, the detrital zircon ages of the supracrustal rocks (Barr et al. 2003; Samson et al. 2005) differ from those of the Carrabassett Formation. In contrast, the detrital zircon ages of the Carrabassett Formation are a good match for Ganderian ages. Thus the only potential source that matches all measured characteristics of the Carrabassett Formation is Ganderia.

A Ganderian provenance is in apparent contrast to tectonic scenarios which indicate that Ganderia was already accreted to Laurentia ( 430 Ma, van Staal et al. 2004, 2006), apparently serving as basement upon which the sediments of the Central Maine Basin, including the Carrabassett Formation, were deposited. However, the eastern margin of Ganderia may have been exposed in the early Devonian if crustal-scale wedging of Ganderia by Avalonia as proposed for eastern Newfoundland (van der Velden et al. 2004) also occurred in northern New England. The likelihood of this scenario is increased by the identification of similar wedging along the Ganderia-Avalonia boundary in southern New England (Aleinikoff et al. 2007; Walsh et al. 2007).

We envision the wedging of Ganderian crust by the Avalonian indenter to have initiated with the Salinic orogeny in the early to middle Silurian. The closure around Ganderia would have lead to interference among the lithospheric mantles of Laurentia, Ganderia, and Avalonia, apparently resulting in delamination of the lithospheric mantle of Ganderia. During early stages of delamination the mantle likely created a keel, causing subsidence of the basin. To the east, where Ganderian and Avalonian crusts interacted, wedging caused slices of Ganderia to override Avalonia (Wintsch et al. 1992). The consequent crustal thickening created relief (Fig. 12). Erosion and transportation yielded sediments with westward paleocurrent directions (Bradley and Hanson 1989, 2002) and geochemical signatures and a detrital zircon suite consistent with a Ganderian passive margin source. Potential specific Ganderian source regions include the Miramichi, a known Ganderian highland at that time (Pickerill and Fyffe 1999), and the Liberty-Orrington and St. Croix sequences (van Staal et al. 1996; Tucker et al. 2001; Reusch et al. 2006).

\section{Provenance and tectonic setting of the Kittery Formation}

Wintsch et al. (2007) noted that the Kittery Formation contains primarily Mesoproterozoic detrital zircons; only two of their analyses ware younger. Paleocurrent directions from the east and the absence of a significant Ordovician age population in the formation led Wintsch and coworkers to suggest that the formation was derived from peri-Gondwanan sources and deposited in the Fredericton Sea, farther east of the Miramichi arch (Tucker et al. 2001). A Fredericton Sea origin for the Kittery sediments could explain the differences in the relative probability plots of the Carrabassett and Kittery formations shown in Fig. 10.

\section{CONCLUSIONS}

The Carrabassett Formation of western Maine is the youngest, most widespread pre-Acadian formation of the Central Maine Basin. The sediments were deposited in a northwestmigrating trench slope during the initial stages of the Acadian orogeny and represent a pulse of sediment derived from Ganderia to the east. The detrital zircons in the Carrabassett Formation are most compatible with chronological fingerprints of Ganderia, specifically the dominant population of ages of $\sim 600 \mathrm{Ma}$ and 900-1440 Ma. Both the Carrabassett

\begin{tabular}{|c|c|c|c|c|c|}
\hline & $\begin{array}{l}\text { Paleocurrent } \\
\text { Indicators }\end{array}$ & $\begin{array}{l}\text { Bulk-rock } \\
\text { Major/Trace } \\
\text { Elements }\end{array}$ & $\begin{array}{l}\text { Bulk-rock } \\
\text { Nd Isotopes }\end{array}$ & $\begin{array}{l}\text { Nd Model } \\
\text { Ages }\end{array}$ & $\begin{array}{l}\text { Detrital } \\
\text { Zircons }\end{array}$ \\
\hline Ganderia & $X$ & $X$ & $X$ & $X$ & $X$ \\
\hline $\begin{array}{l}\text { Juvenile } \\
\text { Avalonia }\end{array}$ & $X$ & 一 & - & - & 一 \\
\hline $\begin{array}{c}\text { Avalonia } \\
\text { Supracrustals }\end{array}$ & $X$ & $X$ & $X$ & $X$ & 一 \\
\hline Laurentia & - & $X$ & $X$ & $X$ & - \\
\hline
\end{tabular}

Fig. 11. Matrix diagram showing matches between the bulk-rock major and trace element characteristics, bulk-rock Nd isotopic values, $\mathrm{Nd}$ model ages, and detrital zircon ages of the Carrabassett Formation and Ganderia. Carrabassett Formation paleocurrent indicators reflect an outboard source. Although some of these parameters also match other terranes, one or more mismatches complicate either Avalonia or Laurentia as sources. 


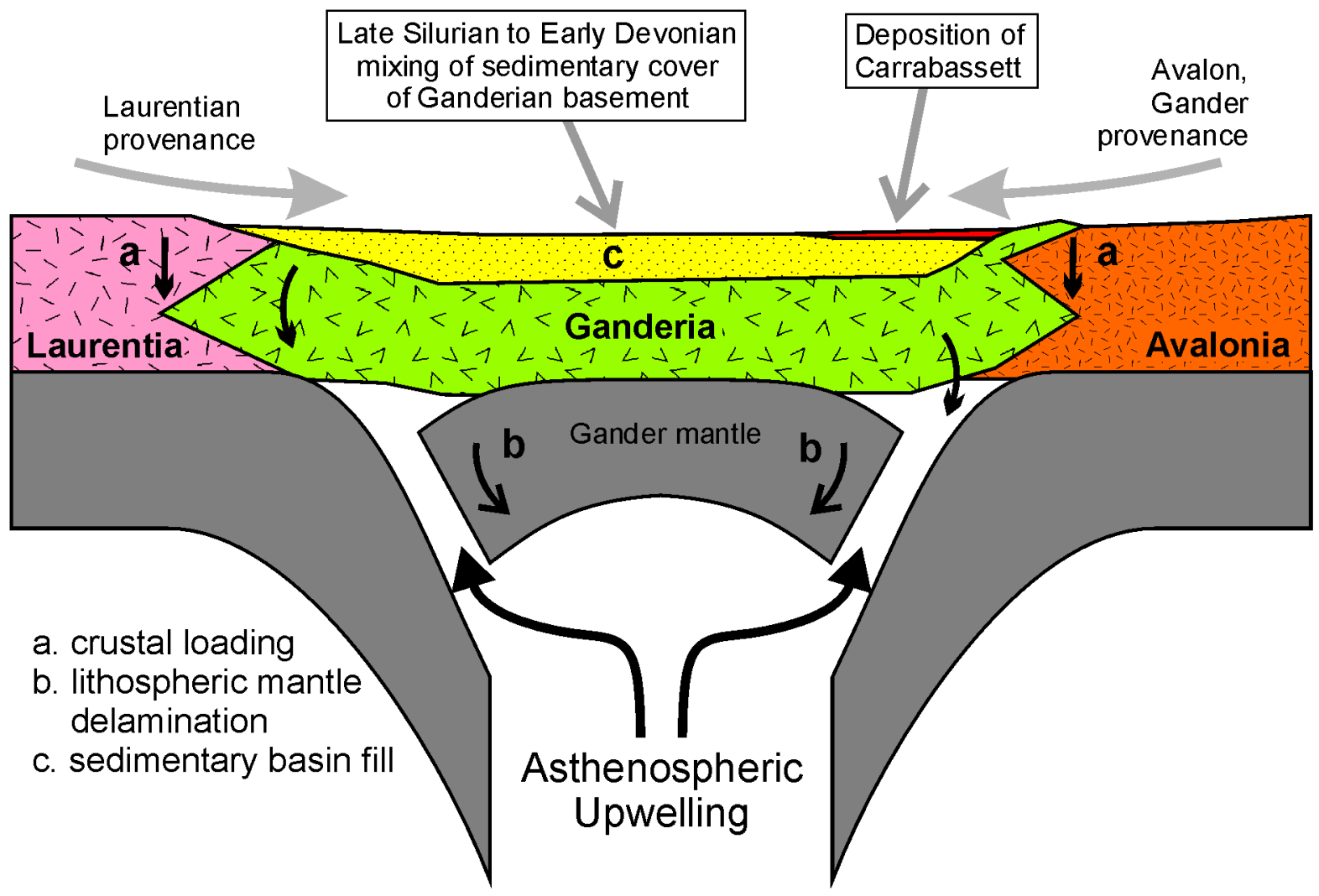

Fig. 12. A schematic diagram showing tectonic wedging of the Ganderian and Avalonian plates to produce the highlands on the Ganderia terrane. The Carrabassett Formation probably represents turbidites shed from these highlands.

Formation and Ganderia have zircons with ages of $\sim 600 \mathrm{Ma}$ and between 2000-2400 Ma, ages that are rare to absent in Laurentia and Avalonia, respectively. REE abundances and $\mathrm{Zr} / \mathrm{Sc}$ versus $\mathrm{Th} / \mathrm{Sc}$ ratios suggest trailing edge turbidites for the Carrabassett and Kittery formations, reflecting the passive margin setting of Ganderia.

Carrabassett Formation bulk-rock $\varepsilon_{\mathrm{Nd}(413 \mathrm{Ma})}$ values are negative (-8 to -10), unlike the positive values for juvenile Avalonian crust. Similar negative values for Laurentia-derived sediments in the Central Maine Basin (Rangeley Formation) and periGondwana-derived Merrimack Trough sediments indicate the inability of bulk-rock $\varepsilon_{\mathrm{Nd}}$ values to discriminate Laurentian and Gondwanan sources when sediments were derived form large regions of mature crust. Unless the sedimentary source is restricted to and dominated by juvenile arc detritus such as Avalonian crust, mixing of sediment yields an intermediate crustal composition, as does bulk-rock incompatible/compatible element ratios.

Even though Ganderia was basement to the sediments of the Central Maine Basin, tectonic wedging of Avalonia into Ganderia is inferred to have uplifted Ganderia outboard of the Central Maine Basin, permitting shedding of Ganderiaderived sediments to the west as the first outboard pulse of sediment. We infer that the collision of Avalonia with the Taconic- and Salinic-modified Laurentian margin did indeed cause the Acadian orogeny in New England, but because the leading edge of Avalonia wedged mid-crustal Ganderia, the portion of Avalonia that experienced Acadian metamorphism lies beneath Ganderian rocks in eastern New England.

\section{ACKNOWLEDGMENTS}

We thank Dwight Bradley for a helpful review of an earlier version of the manuscript, Cees van Staal for many helpful discussions, and two anonymous reviewers and Sandra Barr whose suggestions contributed to improving the manuscript. Initial research funds were provided by the College of Physical and Mathematical Sciences of Brigham Young University, followed by National Science Foundation (EAR-0510857) funds to Robert P. Wintsch and Michael J. Dorais.

\section{REFERENCES CITED}

Ague, J.J. 1991. Evidence for major mass transfer and volume strain during regional metamorphism of pelites. Geology, 19, pp. 855-858. doi:10.1130/0091-7613(1991)019<0855:EF MMTA $>2.3 . \mathrm{CO} ; 2$

Ague, J.J. 1997. Composional variations in metamorphosed sediments of the Littleton Formation, New Hamsphire: Discussion. American Journal of Science, 297, pp. 440-449. 
Aleinikoff, J.N., Zartman, R.E., and Lyons, J.B. 1979. U-Th-Pb geochronology of the Massabesic Gneiss and the granite near Milford, south-central New Hampshire: New evidence for Avalonian basement and Taconic and Alleghenian disturbance in eastern New England. Contributions to Mineralogy and Petrology, 71, pp. 1-11. doi:10.1007/BF00371877

Aleinikoff,J.N., Walter, M., and Fanning, C.M. 1995. U-Pb ages of zircon, monazite, and sphene from rocks of the Massabesic Gneiss complex and Berwick Formation, New Hampshire and Massachusetts. Geological Society of America Abstracts with Programs, 27, p. 26.

Aleinikoff, J.N., Wintsch, R.P., Tollo, R.P., Unruh, D.M., Fanning, C.M., and Schmitz, M.D. 2007. Ages and origins of rocks of the Killingworth dome, south-central Connecticut: Implications for the tectonic evolution of southern New England. American Journal of Science, 307, pp. 63-118. doi:10.2475/01.2007.04

Allegre, C.J., and Rousseau, D. 1984. The growth of the continent through time studied by $\mathrm{Nd}$ isotopic analyses of shales. Earth and Planetary Science Letters, 67, pp. 19-34. doi:10.1016/0012-821X(84)90035-9

Anderson, C.B., and Samson, S.D. 1995. Temporal changes in $\mathrm{Nd}$ isotopic composition of sedimentary rocks in the Sevier and Taconic foreland basins: Increasing influence of juvenile sources. Geology, 23, pp. 983-986. doi:10.1130/00917613(1995)023<0983:TCINIC >2.3.CO;2

Barr, S.M., Davis, D.W., Kamo, S., and White, C.E. 2003. Significance of $\mathrm{U}-\mathrm{Pb}$ detrital zircon ages in quartzite from peri-Gondwanan terranes, New Brunswick and Nova Scotia, Canada. Precambrian Research, 126, pp. 123-145. doi:10.1016/S0301-9268(03)00192-X

Bhatia, M.R., and Crook, K.A.W. 1986. Trace element characteristics of graywackes and tectonic setting discrimination of sedimentary basins. Contributions to Mineralogy and Petrology, 92, pp. 181-193. doi:10.1007/BF00375292

Billings, M.P. 1956. The geology of New Hampshire. Part II. Bedrock Geology: New Hampshire State Planning and Development Committee, Concord, N.H. 200 p.

Bock, B., McLennan, S.M., and Hanson, G.N. 1998. Geochemistry and provenance of the Middle Ordovician Austin Glen Member (Normanskill Formation) and the Taconian orogeny in New England. Sedimentology, 45, pp. 635-655. doi:10.1046/j.1365-3091.1998.00168.x

Bock, B., Hurowitz, J.A., McLennan, S.M., and Hanson, G.N. 2004. Scale and timing of Rare Earth Element redistribution in the Taconian foreland of New England. Sedimentology, 51, pp. 885-897. doi:10.1111/j.1365-3091.2004.00656.x

Bothner, W.A. 1989. Day 6; geology of southern and coastal New Hampshire. In Metamorphism and tectonics of eastern and central North America; Volume 2, A transect through the New England Appalachians. Edited by J.B. Lyons, W.A Bothner, B.L. Doolan, N.L. Jr. Hatch, R.H. Moench, and R.S. Stanley. American Geophysical Union, Washington, DC, United States, pp. 59-64.

Bothner, W.A., and Hussey, A.M. II. 1999. Norumbega connec- tions: Casco Bay, Maine, to Massachusetts? In Norumbega Fault System of the Northern Appalachians. Edited by A. Ludman, and D.P. Jr. West. Geological Society of American Special Paper 331, pp. 59-72.

Bothner, W.A., Boudette, E.L., Fagan, T.J., Gaudette, H.E., Laird, J., and Olszewski, W.J. 1984. Geologic framework of the Massabesic Anticlinorium and the Merrimack Trough, southeastern New Hampshire. In Geology of the Coastal Lowlands, Boston, Mass., to Kennebunk, Maine. Edited by L.S. Hanson. New England Intercollegiate Geological Conference Guidebook, pp. 186-206.

Bradley, D.C., and Hanson, L.S. 1989. Melanges and turbidite facies of the Madrid Formation, Central Maine. In Guidebook for Field Trips in Southern and West-Central Maine. Edited by A.W. Jr. Bery. New England Intercollegiate Geological Congress, $81^{\text {st }}$ Annual Meeting, Farmington, Maine, pp. 183-199.

Bradley, D.C., and Hanson, L.S. 2002. Paleocurrent analysis of a deformed Devonian foreland basin in the northern Appalachians, Maine, USA. Sedimentary Geology, 148, pp. 425447. doi:10.1016/S0037-0738(01)00161-0

Bradley, D.C., Tucker, R.D., Lux, D.R., Harris, A.G., and McGregor, D.C. 2000. Migration of the Acadian orogeny and foreland basin across the northern Appalachians of Maine and adjacent areas. United States Geological Survey Professional Paper 1624, 49 p.

Cox, R.A., Low, D.R., and Cullers, R.L. 1995. The influence of sediment recycling and basement composition on evolution of mudrock chemistry in the southwestern United States. Geochimica Cosmochimica Acta, 59, pp. 29192940. doi:10.1016/0016-7037(95)00185-9

Cox, R.A., Wilton, D.H.C., and Kosler, J. 2003. Laser-ablation U-Th-Pb in situ dating of zircon and allanite: an example from the October Harbor granite, central coastal Labrador, Canada. Canadian Mineralogist, 41, pp. 273-291. doi:10.2113/gscanmin.41.2.273

Cullers, R.L. 1994. The controls on the major and trace element variations of shales, siltstones, and sandstones of Pennsylvania-Permian ages from uplifted continental blocks in Colorado to platform sediments in Kansas. Geochimica Cosmochimica Acta, 58, pp. 4955-4972. doi:10.1016/00167037(94)90224-0

Cullers, R.L., Chaudhuri, S., Arnold, B., Lee, M., and Wolf, C.W. 1975. Rare-earth distributions in clay minerals and clay-sized fractions of Lower Permian Havensville and Eskridge shales of Kansas and Oklahoma. Geochimica Cosmochimica Acta, 39, pp. 1691-1703. doi:10.1016/00167037(75)90090-3

Cullers, R.L., Chaudhure, S., Kilbane, N., and Koch, R. 1979. Rate earths in size fractions and sedimentary rocks of Pennsylvania-Permian age from the mid-continent of the USA. Geochimica Cosmochimica Acta, 43, pp. 1285-1302. doi:10.1016/0016-7037(79)90119-4

Cullers, R.L., Bock, B., and Guidotti, C. 1997. Elemental distributions and neodymium isotopic compositions of Silurian 
metasediments, western Maine, USA: Redistribution of the rare earth elements. Geochimica Cosmochimica Acta, 61, pp. 1847-1861. doi:10.1016/S0016-7037(97)00048-3

Dallmeyer, R.D., and Takasu, A. $1992 .{ }^{40} \mathrm{Ar} /{ }^{39} \mathrm{Ar}$ ages of detrital muscovite and whole-rock slate/phyllite, Narragansett Basin, RI-MA, USA: Implications for rejuvenation during very low-grade metamorphism. Contributions to Mineralogy and Petrology, 110, pp. 515-527. doi:10.1007/BF00344085

Dorais., M.J., Wintsch, R.P., Kunk, M.J., Aleinikoff, J.N., Underdown, C., and Kerwin, C.M. 2007. Pressure-temperature-time conditions, $\mathrm{Nd}$ and $\mathrm{Pb}$ isotopic compositions and detrital zircon geochronology of the Massabessic Gneiss Complex, New Hampshire. Geological Society of America Abstracts with Programs, 40, p. 14.

Eusden,J.D. 1988. Stratigraphy, structure, and metamorphism of the 'Dorsal Zone', central New Hampshire. In Guidebook for field trips in southwestern New Hampshire, southeastern Vermont, and northcentral Massachusetts. Edited by W.A. Bothner. New England Intercollegiate Geological Congress, $80^{\text {th }}$ Annual Meeting, Keene, N.H. pp. 40-59.

Eusden, J.D., and Barreiro, B. 1988. The timing of peak highgrade metamorphism in central-eastern New England. Atlantic Geology, 24, pp. 241-255.

Eusden, J.D., Guzofski, C.A., Robinson, A.G., and Tucker, R.D. 2000. Timing of the Acadian orogeny in northern New Hampshire. Journal of Geology, 108, pp. 219-231. doi: $10.1086 / 314396$

Getty, S.R., and Gromet, L.P. 1992. Geochemical constraints on ductile deformation, crustal extension, and doming about a basement-cover boundary, New England Appalachians. American Journal of Science, 292, pp. 359-379.

Gleason, J.D., Patchett, P.J., Dickinson, W.R., and Ruiz, J. 1994. Nd isotopes link Ouachita turbidites to Appalachian sources. Geology, 22, pp. 347-350. doi:10.1130/00917613(1994)022<0347:NILOTT>2.3.CO;2

Goldstein, S.L., and Jacobsen, S.B. 1988. Nd and Sr isotopic systematics of river water suspended material; implications for crustal evolution. Earth and Planetary Science Letters, 87, pp. 249-265. doi:10.1016/0012-821X(88)90013-1

Goldstein, S.L., O’Nions, R.K., and Hamilton, P.J. 1984. A Sm$\mathrm{Nd}$ isotopic study of atmospheric dusts and particulates from major river systems. Earth and Planetary Science Letters, 70, pp. 221-236. doi:10.1016/0012-821X(84)90007-4

Goldstein, S.L., Arndt, N.T., and Stallard, R.F. 1997. The history of a continent from $\mathrm{U}-\mathrm{Pb}$ ages of zircon from Orinoco River and Sm-Nd isotopes in Orinoco basin river sediments. Chemical Geology, 139, pp. 271-286. doi:10.1016/S00092541(97)00039-9

Hanson, L.S., and Bradley, D.C. 1989. Sedimentary facies and tectonic interpretation of the Carrabassett Formation, north-central Maine. In Structure and Stratigraphy: Studies in Maine Geology. Edited by R.G. Marvinney and R.D. Tucker. Maine Geological Survey, 1, pp. 101-126.

Hanson, L.S., Bradley, D.C., and Caldwell, D.W. 1993. Geology and geomorphology of the Acadian orogen, central Maine.
In Guidebook for Field Trips in the Northeastern United States. Edited by J.T. Cheney and J.C. Hepburn. Geological Society of America Annual Meeting in Boston, Department of Geology and Geography, University of Massachusetts, Contribution No. 67-2, pp. CC-1 to CC-27.

Hatch, N.L., and Moench, R.H. 1984. Bedrock geologic map of the wilderness and roadless areas of the White Mountain National Forest, Coos, Carroll, and Grafton counties, New Hampshire. United States Geological Survey Miscellaneous Map Series, scale 1:125 000.

Hatch, N.L., Moench, R.H., and Lyons, J.B. 1983. SilurianLower Devonian stratigraphy of eastern and south-central New Hampshire: Extensions from western Maine. American Journal of Science, 283, pp. 739-761.

Herron, M.M. 1988. Geochemical classification of terrigenous sands and shales from core or log data. Journal of Sedimentary Petrology, 58, pp. 820-829.

Hibbard, J.C., van Staal, C.R., Rankin, D.W., and Williams, H. 2006. Lithotectonic map of the Appalachian Orogen, Canada-United States of America. Geological Survey of Canada, Map 2096A, scale 1:1 500000.

Katz, F.J. 1917. Stratigraphy in southwestern Maine and southeastern New Hampshire. United States Geological Survey Professional Paper 108, pp. 165-177.

Keppie, J.D., Davis, D.W., and Krogh, T.E. 1998. U-Pb geochronological constraints on Precambrian stratified units in the Avalon composite terrane of Nova Scotia, Canada: tectonic implications. Canadian Journal of Earth Sciences 35, 222-236. doi:10.1139/cjes-35-3-222

Košler, J., Fonneland, H., Sylvester, P., Tubrett, M., and Pederson, R-B. 2002. U-Pb dating of detrital zircons for sedimentary provenance studies - a comparison of laser ablation ICPMS and SIMS techniques. Chemical Geology, 182, pp. 605-618. doi:10.1016/S0009-2541(01)00341-2

Lathrop, A.S., Blum, J.D., and Chamberlain, C.P. 1996, Nd, Sr and $\mathrm{O}$ isotopic study of the petrogenesis of two syntectonic members of the New Hampshire plutonic series: Contributions to Mineralogy and Petrology, 124, pp. 126-138. doi:10.1007/s004100050180

Ludman, A. 1976. A fossil-based stratigraphy in the Merrimack synclinorium, central Maine. In Contributions to the Stratigraphy of New England. Edited by L.R. Page. Geological Society of America Memoir 148, pp. 65-78.

Ludman, A., and Griffin, J.R. 1974. Stratigraphy and structure of central Maine. In Guidebook for Field Trips in East-Central and North-Central Maine. Edited by P.H. Osberg. New England Intercollegiate Geological Congress, $66^{\text {th }}$ Annual Meeting, Orono, Maine, pp. 154-179.

Ludwig, K.L. 2000. Users manual for Isoplot/Ex v. 2.3. A geochronological toolkit for Microsoft Excel. Berkeley Geochronology Center Special Publication No. 1a, Berkeley, California, $54 \mathrm{p}$.

Lux, D.R., and West, D.P. Jr. 1993. New ${ }^{40} \mathrm{Ar} /{ }^{39} \mathrm{Ar}$ mica ages from eastern New Hampshire and southern Maine: Impli- 
cations for the exhumation history of the region. Geological Society of America Abstracts with Programs, 25, p. 35.

Lyons, J.B. 1988. Geology of the Penacook and Mount Kearsarge quadrangles, New Hampshire. In Guidebook for field trips in southwestern New Hampshire, southeastern Vermont, and northcentral Massachusetts. Edited by W.A. Bothner. New England Intercollegiate Geological Congress, $80^{\text {th }}$ Annual Meeting, Keene, NH pp. 60-69.

McLennan, S.M., Taylor, S.R., McCulloch, M.T., and Maynard, J.B. 1990. Geochemical and Nd-Sr isotopic composition of deep-sea turbidites: Crustal evolution and plate tectonic association. Geochimica Cosmochimica Acta, 54, pp. 20152050. doi:10.1016/0016-7037(90)90269-Q

McLennan, S.M., Hemming, S., McDaniel, D.K., and Hanson, G.N. 1993. Geochemical approaches to sedimentation, provenance, and tectonics. In Processes Controlling the Composition of Clastic Sediments. Edited by M.J. Johnson and A. Basu. Geological Society of America Special Paper 284, pp. 21-40.

Michard, A., Gurriet, P., Soudant, M., and Albarede, F. 1985. Nd isotopes in French Phanerozoic shales: external vs. internal aspects of crustal evolution. Geochimica Cosmochimica Acta, 49, pp. 601-610. doi:10.1016/0016-7037(85)90051-1

Moench, R.H., and Aleinikoff, J.N. 2003. Stratigraphy, geochronology, and accretionary terrane settings of two Bronson Hill arc seuences, northern New England. Physics and Chemistry of the Earth, 28, pp. 113-160.

Moench, R.H., and Boudette, E.L. 1970. Stratigraphy of the northwest limb of the Merrimack synclinorium in the Kennebago Lake, Rangeley and Phillips quadrangles, western Maine. In Guidebook for Field Trips in the Rangeley Lakes - Dead River Basin, Western Maine. Edited by G,M. Boone. New England Intercollegiate Geological Conference, $62^{\text {nd }}$ Annual Meeting, Rangely, Maine, pp. 1-25.

Moench, R.H., and Pankiwskyj, K.A. 1988. Geologic map of western Maine.U.S. Geological Survey Miscellaneous Investigations Map 1-1692, scale 1:250 000.

Mosher, S., Murray, D.P., Hermes, O.D., and Gromet, P.L. 1993. Alleghanian and Avalonian tectonism in southeast New England. In Guidebook for the Northeastern United States: Field Trip 1993 Boston. Edited by J.T. Cheney and J.C. Hepburn. Geological Society of America, v. 2, pp. BB1BB30.

Murphy, J.B. 2002. Geochemistry of the Neoproterozoic metasedimentary Gamble Brook Formation, Avalon terrane, Nova Scotia: Evidence for a rifted-arc environment along the west Gondwanan margin of Rodinia. Journal of Geology, 110, pp. 407-419. doi:10.1086/340630

Murphy, J.B., and Nance, R.D. 2002. Sm-Nd isotopic systematics as tectonic tracers: an example from West Avalonia in the Canadian Appalachians. Earth Science Reviews, 59, pp. 77-100. doi:10.1016/S0012-8252(02)00070-3

Murphy, J.B., Fernández-Suárez, J., Jeffries, T.E., and Strachan, R.A. 2004. U-Pb (LA-ICP-MS) dating of detrital zircons from Cambrian clastic rocks in Avalonia: erosion of a
Neoproterozoic arc along the northern Gondwanan margin. Journal of the Geological Society, London, 161, 243254. doi:10.1144/0016-764903-064

Nance, R.D., and Murphy, J.B. 1994. Contrasting basement isotopic signatures and the palinspastic restoration of peripheral orogens: Example from the Neoproterozoic Avalonian-Cadomain belt. Geology, 22, pp. 617-620. doi:10.1130/0091-7613(1994)022<0617:CBISAT>2.3.CO;2

Nance, R.D., Murphy, J.B., and Keppie, J.D. 2002. A Cordilleran model for the evolution of Avalonia. Tectonophysics, 352, pp. 11-31.doi:10.1016/S0040-1951(02)00187-7

O’Nions, R.K., Hamilton, P.J., and Hooker, P.J. 1983. A Nd isotope investigation of sediments related to crustal development in the British Isles. Earth and Planetary Science Letters, 63, pp. 229-240. doi:10.1016/0012-821X(83)90039-0

Osberg, P.H., Tull, J.F., Robinson, P., Hon, R., and Butler, J.R. 1989. The Acadian orogen. In The Appalachian-Ouachita Orogen in the United States. Edited by R.D. Hatcher, W.A. Thomas, and G.W. Viele. The Geological Society of America, The Geology of North America, Volume F-2, pp. 179-232.

Pickerill, R.K. and Fyffe, L.R. 1999. The stratigraphic significance of trace fossils from the Lower Paleozoic Baskahegan Lake Formation near Woodstock, west-central New Brunswick. Atlantic Geology 35, 205-214.

Pollock, J.C., Wilton, D.H.C., van Staal, C.R., and Morrissey, K.D. 2007. U-Pb detrital zircon geochronological constraints on the Early Sillurian collision of Ganderia and Laurentia along the Dog Bay Line: The terminal Iapetan suture in the Newfoundland Appalachians. American Journal of Science 307, 399-433. doi:10.2475/02.2007.04

Rankin, D.W. 1994. Continental margin of the eastern United States: Past and present. In Phanerozoic evolution of North American Continent-Ocean Transitions. Edited by R.C. Speed. Geological Society of America, Decade in North American Geology, Continent-Ocean Transect Volume. pp. 129-218.

Reusch, D.N., van Staal, C., and Hibbard, J. 2006. A central Maine perspective on the Cambro-Orodovician Gander terrane. Geological Society of America Abstracts with Programs, 32, p. 72.

Richerich, S.F. 1983. Sedimentology, stratigraphy, and structure of the Kittery Formation in the Portsmouth, New Hampshire, area. Unpublished M.Sc. thesis, University of New Hampshire, Durham, New Hamphire, 115 p.

Robinson, P., Tucker, R.D., Bradley, D., Berry, H.N. IV., and Osberg, P.H. 1998. Paleozoic orogens in New England, USA. GFF., 120, pp. 119-148.

Roddick, J.C., and Bevier, M.L. 1995. U-Pb dating of granites with inherited zircon: Conventional and ion microprobe results from two Paleozoic plutons, Canadian Appalachians. Chemical Geology, 119, pp. 307-329. doi:10.1016/00092541(94)00107-I

Roy, D.C., and Skehan, J.W.(Editors). 1993. The Acadian Orogeny: Recent studies in New England, Maritime Canada, and 
the autochthonous foreland. Geological Society of America Special Paper 275, 171 p.

Samson, S.D., Barr, S.M., and White, C.E. 2000. Nd isotopic characteristics of terranes within the Avalon Zone, southern New Brunswick. Canadian Journal of Earth Sciences, 37, pp. 1039-1052. doi:10.1139/cjes-37-7-1039

Samson, S.D., D’Lemos, R.S., Miller, B.V., and Hamilton, M.A. 2005. Neoproterozoic palaeogeography of the Cadomia and Avalon terranes: constraints from detrital zircon U-Pb ages. Journal of the Geological Society of London, 162, pp. 6571. doi:10.1144/0016-764904-003

Satkoski, A., Barr, S., and Samson, S. 2007. Sm-Nd isotopic and whole rock chemical compositions of Late Neoproterozoic and Cambrian sedimentary and metasedimentary rocks of the Caledonian highlands (Avalonia), southern New Brunswick. Geological Society of America Abstracts with Programs, 39, p. 95.

Schofield, D.I., and D'Lemos, R.S. 2000. Granite petrogenesis in the Gander zone, NE Newfoundland: mixing of melts from multiple sources and the role of lithospheric delamination. Canadian Journal of Earth Sciences 37, 535-547. doi:10.1139/cjes-37-4-535

Spear, F.S., and Harrison, T.M. 1989. Geochronological studies in central New England I: Evidence for pre-Acadian metamorphism in eastern Vermont. Geology, 17, pp. 181-184. doi:10.1130/0091-7613(1989)017<0181:GSICNE >2.3.CO;2

Taylor, R.S., and McLennan, S.M. 1985. The Continental Crust: Its Composition and Evolution. Blackwell, Oxford, $312 \mathrm{p}$.

Tucker, R.D., and Robinson, P. 1990. Age and setting of the Bronson Hill magmatic arc: a re-evaluation based on U-Pb zircon ages in southeastern New England. Geological Society of America Bulletin, 102, pp. 1404-1419. doi:10.1130/00167606(1990)102<1404:AASOTB >2.3.CO;2

Tucker, R.D., Osberg, P.H., and Berry, H.N. IV. 2001. The geology of a part of Acadia and the nature of the Acadian orogeny across central and eastern Maine. American Journal of Science, 301, pp. 205-260. doi:10.2475/ajs.301.3.205

van der Pluijm, B.A., and van Staal, C.R. 1988. Characteristics and evolution of the central mobile belt, Canadian Appalachians. Journal of Geology 96, 535-547.

van der Velden, A.J., van Staal, C.R., and Cook, F.A. 2004. Crustal structure, fossil subduction, and the tectonic evolution of the Newfoundland Appalachians: Evidence from a reprocessed seismic reflection survey. Geological Society of America Bulletin 116, 1485-1498. doi:10.1130/B25518.1

Van Staal, C.R. 2007. Pre-Carboniferous tectonic evolution and metallogeny of the Canadian Appalachians. In Mineral Deposits of Canada: a synthesis of major deposit-types, district metallogeny, the evolution of geological provinces, and exploration methods. Edited by W.D. Goodfellow. Geological Association of Canada, Mineral Deposits Division, Special Publication 5, pp. 793-818.

van Staal, C.R., Sullivan, R.W, and Whalen, J.B. 1996. Provenance and tectonic histort of the Gander zone in the Caledonian/Appalachian orogen: Implications for the origin and assembly of Avalon. In Avalonian and related periGondwanan terranes of the circum-North Atlantic. Edited by R.D. Nance and M.D. Thompson. Geological Society of America Special Paper 304, pp. 347-367.

van Staal, C.R., McNicoll, V., Valverde-Vaquero, P., Barr, S.M., Fyffe L.R., and Reusch, D.N. 2004. Ganderia, Avalonia, and the Salinic and Acadian orogenies. Geological Society of America Abstracts with Programs, 36, p. 128.

van Staal, C., Lissenberg, J., Rogers, N., McNicoll., V., Ververde, P., Whalen, J., and Zagorevski, A. 2006. The northern Appalachians: an accretionary orogen. Geophysical Research Abstracts, 8, p. 00915.

Walsh, G.J., Aleinikoff, J.N., and Wintsch, R.P. 2007. Origin of the Lyme dome and implications for the timing of multiple Alleghanian deformational and intrusive events in southern Connecticut. American Journal of Science, 307, pp. 168215. doi:10.2475/06.2007.06

West, D.P. Jr. 1993. The eastern limit of Acadian high-grade metamorphism in northern New England: Implications for the location of the "Acadian Sutire". Geological Society of America Abstracts with Programs, 25, p. 89.

Whalen, J.B., Jenner, G.A., Longstaffe, F.J., and Hegner, E. 1996. Nature and evolution of the eastern margin of Iapetus: geochemical and isotopic constraints from Siluro-Devonian granitoid plutons in the New Brunswick Appalachians. Canadian Journal of Earth Sciences 33, 140-155.

Whalen, J.B., van Staal., C.R., Longstaffe, F.J., Gariépy, C., and Jenner, G.A. 1997. Insights into tectonostratigraphic zone identification in southwestern Newfoundland based on isotopic $(\mathrm{Nd}, \mathrm{O}, \mathrm{Pb})$ and geochemical data. Atlantic Geology 33, 231-241.

Wiedenbeck, M., Allé, P., Corfu, F., Griffin, W.L., Meier, M., Oberli, F., von Quadt, A., Roddick, J.C., and Spiegel, W. 1995. Three natural zircon standards for U-Th-Pb, Lu-Hf, trace element and REE analyses. Geostandards Newsletter 19, pp. 1-23. doi:10.1111/j.1751-908X.1995.tb00147.x

Williams, H. 1979. Appalachian Orogen in Canada. Canadian Journal of Earth Sciences 16, 792-807.

Wintsch, R.P., and Sutter, J.F. 1986. A tectonic model for the late Paleozoic of southeastern New England. Journal of Geology, 94, pp. 459-472.

Wintsch, R.P., Sutter, J.F., Kunk, M.J., Aleinikoff, J.N., and Dorais, M.J. 1992. Contrasting P-T-t paths: Thermochronologic evidence for a Late Paleozoic final assembly of the Avalon composite terrane in the New England Appalachians. Tectonics, 11, pp. 672-689. doi:10.1029/91TC02904

Wintsch, R.P., Sutter, J.F., Kunk, M.J., Aleinikoff, J.N., and Boyd, J.L. 1993. Alleghanian assembly of Proterozoic and Paleozoic lithotectonic terranes in south central New England: New constraints from geochronology and petrology. In Field Trip Guidebook for the Northeastern United States: 1993 Boston. Edited by J.T. Cheney and J.C. Hepburn. Geological Society of America, v. 1, pp. H1-H30.

Wintsch, R.P., AKeInikoff, J.N., Walsh, G.J., Bothner, W.A., Jussey, A.M. II, AND FANNING, C.M. 2007. Shrimp U-Pb evi- 
dence for a Late Silurian age of metasedimentary rocks in the Merrimack and Putnam-Nashoba terranes, eastern New England. American Journal of Science, 307, pp. 119-167. doi:10.2475/01.2007.05

WronKIEWICZ, D.J., AND CONDIE, K.C. 1990. Geochemistry and mineralogy of sediments from the Ventersdorp and Transvaal Supergroups, South Africa: Cratonic evolution during the early Proterozoic. Geochimica Cosmochimica Acta, 54, pp. 343-354. doi:10.1016/0016-7037(90)90323-D

ZARTMAN, R.E., AND NAYLOR, R.S. 1984. Structural implications of some radiometric ages of igneous rocks in southeastern New England. Geological Society of America Bulletin, 95, pp. 522-539. doi:10.1130/0016-7606(1984)95<522:SIOSRA $\geq 2.0 . \mathrm{CO} ; 2$

Editorial responsibility: Sandra M. Barr

Appendix A. Sample locations for bulk-rock analyses based on best estimates from Google Earth.

\begin{tabular}{|c|c|c|c|c|c|}
\hline \multicolumn{3}{|c|}{ Carrabassett Formation } & \multicolumn{3}{|c|}{ Eliot Formation } \\
\hline \multicolumn{3}{|c|}{ Sample Latitude } & Sample & Latitude & Longitude \\
\hline $\mathrm{H}-2$ & $45^{\circ} 22^{\prime} 08.69^{\prime \prime} \mathrm{N}$ & $69^{\circ} 26^{\prime} 12.00^{\prime \prime} \mathrm{W}$ & $\overline{\text { E-1 }}$ & $42^{\circ} 52^{\prime} 12.25^{\prime \prime} \mathrm{N}$ & $70^{\circ} 58^{\prime} 41.32^{\prime \prime} \mathrm{W}$ \\
\hline $\mathrm{H}-3$ & $45^{\circ} 22^{\prime} 08.69^{\prime \prime} \mathrm{N}$ & $69^{\circ} 26^{\prime} 12.00^{\prime \prime} \mathrm{W}$ & E-2 & $42^{\circ} 52^{\prime} 12.25^{\prime \prime} \mathrm{N}$ & $70^{\circ} 58^{\prime} 41.32^{\prime \prime W}$ \\
\hline H-5 & $45^{\circ} 18^{\prime} 14.05^{\prime \prime} \mathrm{N}$ & $69^{\circ} 26^{\prime} 09.13^{\prime \prime W}$ & E-3 & $42^{\circ} 52^{\prime} 12.25^{\prime \prime} \mathrm{N}$ & $70^{\circ} 58^{\prime} 41.32^{\prime \prime W}$ \\
\hline H-6 & $45^{\circ} 18^{\prime} 14.05^{\prime \prime N}$ & $69^{\circ} 26^{\prime} 09.13^{\prime \prime W}$ & E-4 & $42^{\circ} 52^{\prime} 12.25^{\prime \prime} \mathrm{N}$ & $70^{\circ} 58^{\prime} 41.32^{\prime \prime W}$ \\
\hline $\mathrm{H}-7$ & $45^{\circ} 18^{\prime} 14.05^{\prime \prime} \mathrm{N}$ & $69^{\circ} 26^{\prime} 09.13^{\prime \prime W}$ & & & \\
\hline $\mathrm{H}-8$ & $45^{\circ} 18^{\prime} 14.05^{\prime \prime} \mathrm{N}$ & $69^{\circ} 26^{\prime} 09.13^{\prime \prime W}$ & \multicolumn{3}{|c|}{ Kittery Formation } \\
\hline $\mathrm{H}-9$ & $45^{\circ} 16^{\prime} 16.01^{\prime \prime N}$ & $69^{\circ} 28^{\prime} 56.98^{\prime \prime W}$ & $\mathrm{~K}-1$ & $43^{\circ} 05^{\prime} 28.42^{\prime \prime} \mathrm{N}$ & $70^{\circ} 51^{\prime} 55.75^{\prime \prime} \mathrm{W}$ \\
\hline $\mathrm{H}-10$ & $45^{\circ} 16^{\prime} 16.01^{\prime \prime} \mathrm{N}$ & $69^{\circ} 28^{\prime} 56.98^{\prime \prime W}$ & K-4 & $43^{\circ} 14^{\prime} 40.28^{\prime \prime} \mathrm{N}$ & $70^{\circ} 35^{\prime} 27.76^{\prime \prime W}$ \\
\hline \multirow[t]{2}{*}{$\mathrm{H}-11$} & $45^{\circ} 22^{\prime} 08.69^{\prime \prime} \mathrm{N}$ & $69^{\circ} 26^{\prime} 12.00^{\prime \prime} \mathrm{W}$ & K-6 & $43^{\circ} 14^{\prime} 40.28^{\prime \prime} \mathrm{N}$ & $70^{\circ} 35^{\prime} 27.76^{\prime \prime W}$ \\
\hline & & & K-9 & $43^{\circ} 17^{\prime} 14.97^{\prime \prime} \mathrm{N}$ & $70^{\circ} 34^{\prime} 12.78^{\prime \prime W}$ \\
\hline \multicolumn{3}{|c|}{ Rangeley Formation } & K-11 & $43^{\circ} 33^{\prime} 30.57^{\prime \prime} \mathrm{N}$ & $70^{\circ} 12^{\prime} 18.45^{\prime \prime} \mathrm{W}$ \\
\hline $\mathrm{R}-2-1$ & $43^{\circ} 27^{\prime} 42.29^{\prime \prime} \mathrm{N}$ & $71^{\circ} 40^{\prime} 19.05^{\prime \prime W}$ & $\mathrm{~K}-12$ & $43^{\circ} 33^{\prime} 30.57^{\prime \prime} \mathrm{N}$ & $70^{\circ} 12^{\prime} 18.45^{\prime \prime W}$ \\
\hline $\mathrm{R}-2-2$ & $43^{\circ} 27^{\prime} 42.29^{\prime \prime} \mathrm{N}$ & $71^{\circ} 40^{\prime} 19.05^{\prime \prime W}$ & & & \\
\hline R-3-1 & $43^{\circ} 27^{\prime} 42.29^{\prime \prime} \mathrm{N}$ & $71^{\circ} 40^{\prime} 19.05^{\prime \prime W}$ & \multicolumn{3}{|c|}{ Berwick Formation } \\
\hline $\mathrm{R}-3-2$ & $43^{\circ} 27^{\prime} 42.29^{\prime \prime} \mathrm{N}$ & $71^{\circ} 40^{\prime} 19.05^{\prime \prime W}$ & B-1 & $42^{\circ} 52^{\prime} 12.25^{\prime \prime} \mathrm{N}$ & $71^{\circ} 20^{\prime} 37.44^{\prime \prime} \mathrm{W}$ \\
\hline $\mathrm{R}-5-1$ & $43^{\circ} 23^{\prime} 00.61^{\prime \prime} \mathrm{N}$ & $71^{\circ} 26^{\prime} 29.31^{\prime \prime W}$ & B-2 & $42^{\circ} 52^{\prime} 10.60^{\prime \prime} \mathrm{N}$ & $71^{\circ} 20^{\prime} 37.07^{\prime \prime} \mathrm{W}$ \\
\hline $\mathrm{R}-5-2$ & $43^{\circ} 23^{\prime} 00.61^{\prime \prime} \mathrm{N}$ & $71^{\circ} 26^{\prime} 29.31^{\prime \prime W}$ & B-3 & $42^{\circ} 52^{\prime} 10.60^{\prime \prime} \mathrm{N}$ & $71^{\circ} 20^{\prime} 37.07^{\prime \prime} \mathrm{W}$ \\
\hline R-6-1 & $43^{\circ} 23^{\prime} 00.61^{\prime \prime} \mathrm{N}$ & $71^{\circ} 26^{\prime} 29.31^{\prime \prime W}$ & B-4 & $42^{\circ} 52^{\prime} 10.60^{\prime \prime} \mathrm{N}$ & $71^{\circ} 20^{\prime} 37.07^{\prime \prime} \mathrm{W}$ \\
\hline $\mathrm{R}-6-2$ & $43^{\circ} 23^{\prime} 00.61^{\prime \prime} \mathrm{N}$ & $71^{\circ} 26^{\prime} 29.31^{\prime \prime W}$ & B-5 & $42^{\circ} 53^{\prime} 59.80^{\prime \prime} \mathrm{N}$ & $71^{\circ} 18^{\prime} 18.43^{\prime \prime W}$ \\
\hline \multirow[t]{2}{*}{$\mathrm{R}-6-3$} & $43^{\circ} 23^{\prime} 00.61^{\prime \prime} \mathrm{N}$ & $71^{\circ} 26^{\prime} 29.31^{\prime \prime W}$ & B-6 & $42^{\circ} 53^{\prime} 59.80^{\prime \prime} \mathrm{N}$ & $71^{\circ} 18^{\prime} 18.43^{\prime \prime W}$ \\
\hline & & & B-8 & $42^{\circ} 58^{\prime} 54.34^{\prime \prime} \mathrm{N}$ & $71^{\circ} 13^{\prime} 17.24^{\prime \prime W}$ \\
\hline \multicolumn{3}{|c|}{ Madrid Formation } & B-9 & $43^{\circ} 00^{\prime} 01.50^{\prime \prime} \mathrm{N}$ & $71^{\circ} 10^{\prime} 33.21^{\prime \prime W}$ \\
\hline $\mathrm{H}-12$ & $45^{\circ} 04^{\prime} 07.44^{\prime \prime} \mathrm{N}$ & $69^{\circ} 54^{\prime} 21.80^{\prime \prime W}$ & & & \\
\hline $\mathrm{H}-13$ & $45^{\circ} 04^{\prime} 06.96^{\prime \prime} \mathrm{N}$ & $69^{\circ} 54^{\prime} 20.99^{\prime \prime W}$ & & & \\
\hline $\mathrm{H}-14$ & $45^{\circ} 04^{\prime} 06.92^{\prime \prime} \mathrm{N}$ & $69^{\circ} 54^{\prime} 20.43^{\prime \prime W}$ & & & \\
\hline $\mathrm{H}-15$ & $45^{\circ} 04^{\prime} 05.74^{\prime \prime} \mathrm{N}$ & $69^{\circ} 54^{\prime} 18.10^{\prime \prime W}$ & & & \\
\hline H-16 & $45^{\circ} 04^{\prime} 05.11^{\prime \prime} \mathrm{N}$ & $69^{\circ} 54^{\prime} 15.63^{\prime \prime W}$ & & & \\
\hline H-19 & $44^{\circ} 57^{\prime} 27.64^{\prime \prime} \mathrm{N}$ & $69^{\circ} 52^{\prime} 13.27^{\prime \prime W}$ & & & \\
\hline $\mathrm{H}-20$ & $44^{\circ} 57^{\prime} 27.64^{\prime \prime} \mathrm{N}$ & $69^{\circ} 52^{\prime} 13.27^{\prime \prime W}$ & & & \\
\hline
\end{tabular}

